\title{
Synthesis of aza-analogues of the glycosylated Tyrosine portion of Mannopeptimycin-E
}

\author{
Sanjeeva R Guppi and George A. O'Doherty* \\ Department of Chemistry, West Virginia University \\ Morgantown, WV 26506 \\ George.ODoherty@mail.wvu.edu
}

Supporting Information

Section A: General Information

S-2

Section B: Experimental Procedures

S-3

Section C: ${ }^{1} \mathrm{H}$ NMR and ${ }^{13} \mathrm{C}$ NMR Spectra

S-13 


\section{Section A: General Information:}

General Methods and materials: ${ }^{1} \mathrm{H}$ and ${ }^{13} \mathrm{C}$ spectra were recorded on 270 and 600 spectrometers. Chemical shifts were reported relative to internal tetramethylsilane $(\delta$ $0.00+)$ or $\mathrm{CDCl}_{3}(\delta 7.26)$ or $\mathrm{CD}_{3} \mathrm{OD}(\delta 4.87)$ for ${ }^{1} \mathrm{H}$ and $\mathrm{CDCl}_{3}(\delta 77.1)$ or $\mathrm{CD}_{3} \mathrm{OD}(\delta$ 49.15) for ${ }^{13} \mathrm{C}$. Optical rotations were measured with a digital polarimeter in the solvent specified. Infrared (IR) spectra were obtained on a FT-IR spectrometer. Flash column chromatography was performed on 60-200 mesh silica gel. Analytical thin-layer chromatography was performed with precoated glass-backed plates and visualized by quenching of fluorescence and by charring after treatment with $p$-anisaldehyde or phosphomolybdic acid or potassium permanganate stain. $R_{f}$ values were obtained by elution in the stated solvent ratios $(\mathrm{v} / \mathrm{v})$. Ether, THF, methylene chloride and triethylamine were dried by passing through activated alumina ( $8 \times 14$ mesh) column with nitrogen gas pressure. Commercial reagents were used without purification unless otherwise noted. Air and/or moisture-sensitive reactions were carried out under an atmosphere of argon/nitrogen using oven/flamed-dried glassware and standard syringe/septa techniques. 


\section{Section B: Experimental Procedures:}

$(1 ' S, 4 ' S, 5 ' R, 1 S, 4 S, 5 R)-1-[1 '-N$-carbobenzyloxy-D-tyrosine methoxycarbonyl -5'(tert-butyl-dimethylsilanyloxymethyl)-1',4'-dihydro-5' H-pyran-4'-yloxy] -5-(tertbutyl-dimethylsilanyloxymethyl)-1,4-dihydro-5 $H$-pyran- $O$-4-methyl carbonate (10):

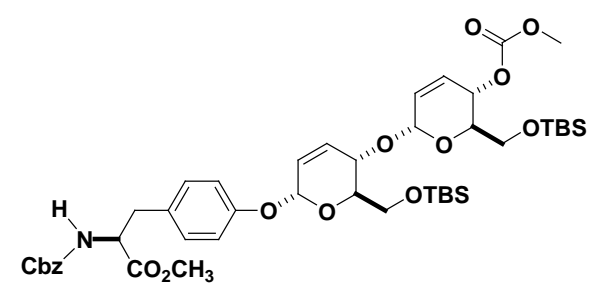

To a solution of allylic alcohol $6(400 \mathrm{mg}, 0.50 \mathrm{mmol})$ in $\operatorname{dry} \mathrm{CH}_{2} \mathrm{Cl}_{2}(1.0 \mathrm{~mL})$ at $0{ }^{\circ} \mathrm{C}$, was added pyridine $(242 \mu \mathrm{L}, 3.00 \mathrm{mmol})$, DMAP (30 mg), and methyl chloroformate (283 mg, $3.00 \mathrm{mmol})$. After stirring $24 \mathrm{~h}$ at room temperature, water $(3 \mathrm{~mL})$ was added and then the mixture was extracted with EtOAc $(3 \mathrm{x} 10 \mathrm{~mL})$, dried $\left(\mathrm{Na}_{2} \mathrm{SO}_{4}\right)$, concentrated under reduced pressure. The crude product was purified using silica gel flash chromatography eluting with EtOAc/hexane (15:85) to give carbonate 10 (402.4 $\mathrm{mg}, 0.45 \mathrm{mmol}, 90 \%)$ as viscous oil. $R_{f}(30 \%$ EtOAc/hexane $)=0.65 ;[\alpha]_{\mathrm{D}}^{26}=+50(c=1$, $\mathrm{CH}_{2} \mathrm{Cl}_{2}$ ); IR (thin film, $\mathrm{cm}^{-1}$ ) 2928, 2885, 1748, 1441, 1509, 1266, 1090, 981, 865; ${ }^{1} \mathrm{H}$ NMR (600 MHz, $\left.\mathrm{CDCl}_{3}\right) \delta 7.34(\mathrm{~m}, 5 \mathrm{H}), 7.00(\mathrm{~m}, 4 \mathrm{H}), 6.18(\mathrm{~d}, J=10.2 \mathrm{~Hz}, 1 \mathrm{H}), 6.01$ $(\mathrm{d}, J=10.2 \mathrm{~Hz}, 1 \mathrm{H}), 5.94(\mathrm{dd}, J=2.4,1.8 \mathrm{~Hz}, 1 \mathrm{H}), 5.92(\mathrm{ddd}, J=10.8,2.4,1.8 \mathrm{~Hz}, 1 \mathrm{H})$, $5.77(\mathrm{ddd}, J=10.2,3.0,1.8 \mathrm{~Hz}, 1 \mathrm{H}), 5.59(\mathrm{~d}, J=1.8 \mathrm{~Hz}, 1 \mathrm{H}), 5.31(\mathrm{dd}, J=3.0,1.8 \mathrm{~Hz}$, $1 \mathrm{H}), 5.29(\mathrm{dd}, J=3.0,2.4 \mathrm{~Hz}, 1 \mathrm{H}), 5.11(\mathrm{~d}, J=12.6 \mathrm{~Hz}, 1 \mathrm{H}), 5.08(\mathrm{~d}, J=12.6 \mathrm{~Hz}, 1 \mathrm{H})$, 4.61 (ddd, $J=8.4,6.0,5.4 \mathrm{~Hz}, 1 \mathrm{H}), 4.43(\mathrm{~d}, J=9.6 \mathrm{~Hz}, 1 \mathrm{H}), 3.88(\mathrm{dd}, J=5.4,2.4 \mathrm{~Hz}$, $1 \mathrm{H}), 3.86(\mathrm{dd}, J=6.0,2.4 \mathrm{~Hz}, 1 \mathrm{H}), 3.84(\mathrm{dd}, J=4.8,1.8 \mathrm{~Hz}, 1 \mathrm{H}), 3.83(\mathrm{dd}, J=4.8,2.4$ $\mathrm{Hz}, 1 \mathrm{H}), 3.81(\mathrm{dd}, J=4.8,1.8 \mathrm{~Hz}, 1 \mathrm{H}), 3.79$ (s, 3H), $3.76(\mathrm{~s}, 1 \mathrm{H}), 3.71$ (s, 3H), 3.07 (ddd, 
$J=8.4,5.4,5.4 \mathrm{~Hz}, 1 \mathrm{H}), 3.05(\mathrm{ddd}, J=7.8,6.0,5.4 \mathrm{~Hz}, 1 \mathrm{H}), 0.89$ (s, 9H), $0.84(\mathrm{~s}, 9 \mathrm{H})$, $0.06(\mathrm{~s}, 3 \mathrm{H}), 0.05(\mathrm{~s}, 3 \mathrm{H}), 0.02(\mathrm{~s}, 3 \mathrm{H}), 0.01(\mathrm{~s}, 3 \mathrm{H}) ;{ }^{13} \mathrm{C} \mathrm{NMR}\left(150 \mathrm{MHz}, \mathrm{CDCl}_{3}\right) \delta$ $171.9,156.6,155.6,155.0,136.2,130.3,130.1$ (2C), 129.4, 129.0, 128.5 (2C), 128.1, $128.0,127.6,126.2,117.2,117.0,109.9,92.9,91.0,71.4,69.3,68.4,66.9,66.4,62.7$, $62.0,54.9,54.8,52.2,37.4,25.93(3 \mathrm{C}), 25.91(3 \mathrm{C}), 18.43,18.42,-5.0,-5.2,-5.4(2 \mathrm{C})$; CIHRMS Calcd for $\left[\mathrm{C}_{44} \mathrm{H}_{65} \mathrm{NO}_{13} \mathrm{Si}_{2} \mathrm{Na}^{+}\right]$: 894.3892. Found 894.3890.

$(1 ' S, 4 ' S, 5 ' R, 1 S, 4 S, 5 R)-1-[1 '-N$-carbobenzyloxy-D-tyrosine methoxycarbonyl -5'-(tertbutyl-dimethylsilanyloxymethyl)-1',4' -dihydro-5' $H$-pyran-4'-yloxy]-5-(tert-butyldimethylsilanyloxymethyl)-4-azido-1,4-dihydro-5H-pyran (5):

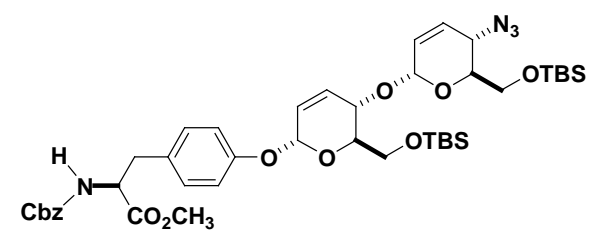

To a mixture of carbonate $\mathbf{1 0}(210 \mathrm{mg}, 0.24 \mathrm{mmol})$, allylpalladium chloride dimer (13.2 $\mathrm{mg}, \quad 0.03 \mathrm{mmol})$ and 1,4-bis(diphenylphosphino)butane $(61.6 \mathrm{mg}, 0.13 \mathrm{mmol})$ in anhydrous THF (0.25 mL) was added $\mathrm{TMSN}_{3}(277 \mathrm{mg}, 1.20 \mathrm{mmol})$ under argon atmosphere. The solution was stirred at room temperature for $3 \mathrm{~h}$. Then the mixture was evaporated under reduced pressure, purified using silica gel flash chromatography eluting with EtOAc/hexane (15:85) to give allylic azide 5 (182 $\mathrm{mg}, 0.21 \mathrm{mmol}, 88 \%)$ as viscous oil. $R_{f}(30 \%$ EtOAc/hexane $)=0.70 ;[\alpha]_{\mathrm{D}}^{26}=+65\left(c=1, \mathrm{CH}_{2} \mathrm{Cl}_{2}\right) ;$ IR (thin film, $\left.\mathrm{cm}^{-1}\right)$ 2952, 2929, 2856, 2103, 1726, 1510, 1253, 1043, 993, 830; ${ }^{1} \mathrm{H}$ NMR (600 MHz, $\left.\mathrm{CDCl}_{3}\right) \delta 7.35(\mathrm{~m}, 5 \mathrm{H}), 6.99(\mathrm{~m}, 4 \mathrm{H}), 6.18(\mathrm{~d}, J=10.2 \mathrm{~Hz}, 1 \mathrm{H}), 6.00(\mathrm{~d}, J=10.2 \mathrm{~Hz}$, 1H), $5.94(\mathrm{ddd}, J=10.8,2.4,1.8 \mathrm{~Hz}, 1 \mathrm{H}), 5.83(\mathrm{ddd}, J=10.2,3.0,2.4 \mathrm{~Hz}, 1 \mathrm{H}), 5.59$ (d, $J$ 
$=2.4 \mathrm{~Hz}, 1 \mathrm{H}), 5.26(\mathrm{~d}, J=1.8 \mathrm{~Hz}, 1 \mathrm{H}), 5.11(\mathrm{~d}, J=12.6 \mathrm{~Hz}, 1 \mathrm{H}), 5.08(\mathrm{~d}, J=12.6 \mathrm{~Hz}$, 1H), $4.62(\mathrm{ddd}, J=7.8,6.0,5.4 \mathrm{~Hz}, 1 \mathrm{H}), 4.42(\mathrm{~d}, J=9.0 \mathrm{~Hz}, 1 \mathrm{H}), 4.14(\mathrm{dd}, J=3.8,1.8$ $\mathrm{Hz}, 1 \mathrm{H}), 4.12(\mathrm{dd}, J=10.2,1.8 \mathrm{~Hz}, 1 \mathrm{H}), 3.92(\mathrm{~d}, J=3.0 \mathrm{~Hz}, 1 \mathrm{H}), 3.90(\mathrm{~d}, J=3.0 \mathrm{~Hz}$, 1H), 3.88 (ddd, $J=4.2,3.6,3.0 \mathrm{~Hz}, 1 \mathrm{H}), 3.86(\mathrm{~d}, J=1.8 \mathrm{~Hz}, 1 \mathrm{H}), 3.80(\mathrm{dd}, J=4.2,2.4$ $\mathrm{Hz}, 1 \mathrm{H}), 3.71$ (s, 3H), 3.65 (ddd, $J=9.0,3.0,1.8 \mathrm{~Hz}, 1 \mathrm{H}), 3.08$ (ddd, $J=8.4,6.0,5.4 \mathrm{~Hz}$, 1H), 3.04 (ddd, $J=8.4,6.0,5.4 \mathrm{~Hz}, 1 \mathrm{H}), 0.91$ (s, 9H), 0.85 (s, 9H), 0.099 (s, 3H), 0.094 (s, 3H), 0.03 (s, 3H), 0.02 (s, 3H); ${ }^{13} \mathrm{C}$ NMR $\left(150 \mathrm{MHz}, \mathrm{CDCl}_{3}\right) \delta 171.9,156.6,155.6$, 136.2, 130.3, 130.1 (2C), $129.0(2 \mathrm{C}), 128.5$ (2C), 128.1, $128.0(2 \mathrm{C}), 126.2,117.2(2 \mathrm{C})$ $109.9,92.9,90.8,71.3,70.5,66.9,66.2,62.6,62.4,54.8,53.4,52.2,37.3,25.9(6 \mathrm{C}), 18.4$ (2C), -5.0, -5.2 (2C), -5.3; CIHRMS Calcd for $\left[\mathrm{C}_{42} \mathrm{H}_{62} \mathrm{~N}_{4} \mathrm{O}_{10} \mathrm{Si}_{2} \mathrm{Na}^{+}\right]$: 861.3902. Found 861.3910.

$(1 ' S, 4 ' S, 5 ' R, 1 S, 4 S, 5 R)-1-[1 '-N \quad$-carbobenzyloxy-D-tyrosine methoxycarbonyl $\quad-5$ '(tert-butyl-dimethylsilanyloxymethyl)-1',4'-dihydro-5' H-pyran-4' -yloxy]-5-(tertbutyl-dimethylsilanyloxymethyl)-4-amino-1,4-dihydro-5H-pyran-(4)

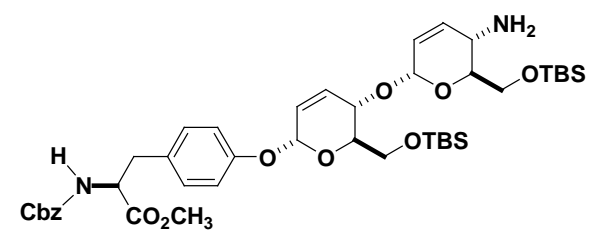

To a solution of Azide $5(140 \mathrm{mg}, 0.16 \mathrm{mmol})$ in $\mathrm{THF} / \mathrm{H}_{2} \mathrm{O}(9: 1$, v/v, $0.3 \mathrm{ml})$ was added $(n-\mathrm{Bu})_{3} \mathrm{P}(84 \mathrm{mg}, 0.41 \mathrm{mmol})$, then the mixture was stirred at room temperature for $1 \mathrm{~h}$. The reaction mixture was evaporated with a little silica gel under reduced pressure and the crude product was purified using silica gel flash chromatography eluting with $\mathrm{MeOH} / \mathrm{EtOAc} / \mathrm{hexane}(10: 40: 50)$ to give allylic amine 4 (104 $\mathrm{mg}, 0.13 \mathrm{mmol}, 80 \%)$ as a 
colorless oil, $R_{f}=0.50(10: 40: 50 \mathrm{MeOH} / \mathrm{EtOAc} / \mathrm{Hexane}) ;[\alpha]_{\mathrm{D}}^{26}=+24(c=1, \mathrm{MeOH})$; IR (thin film, $\mathrm{cm}^{-1}$ ) 3361, 2944, 2833, 1740, 1448, 1374, 1240, 1120, 1040, 981, 847; ${ }^{1} \mathrm{H}$ NMR (600 MHz, CD $\left.{ }_{3} \mathrm{OD}\right) \delta 7.27(\mathrm{~m}, 5 \mathrm{H}), 6.99(\mathrm{~m}, 4 \mathrm{H}), 6.21(\mathrm{~d}, J=9.6 \mathrm{~Hz}, 1 \mathrm{H}), 5.91$ $(\mathrm{d}, J=10.2 \mathrm{~Hz}, 1 \mathrm{H}), 5.84(\mathrm{ddd}, J=10.2,10.2,8.4 \mathrm{~Hz}, 1 \mathrm{H}), 5.69(\mathrm{ddd}, J=11.4,10.2$, $10.2 \mathrm{~Hz}, 1 \mathrm{H}), 5.59$ (d, $J=2.4 \mathrm{~Hz}, 1 \mathrm{H}), 5.22$ (br s, 1H), 5.02 (d, $J=12.6 \mathrm{~Hz}, 1 \mathrm{H}), 4.95$ (d, $J=12.6 \mathrm{~Hz}, 1 \mathrm{H}), 4.36(\mathrm{~m}, 1 \mathrm{H}), 4.27(\mathrm{~d}, J=9.0 \mathrm{~Hz}, 1 \mathrm{H}), 3.91(\mathrm{dd}, J=10.8,4.2 \mathrm{~Hz}, 1 \mathrm{H})$, $3.86(\mathrm{~m}, 1 \mathrm{H}), 3.83(\mathrm{dd}, J=6.0,3.0 \mathrm{~Hz}, 1 \mathrm{H}), 3.80(\mathrm{dd}, J=10.2,4.2 \mathrm{~Hz}, 1 \mathrm{H}), 3.75(\mathrm{dd}, J=$ 10.8, $6.0 \mathrm{~Hz}, 1 \mathrm{H}), 3.65$ (s, 3H), 3.50 (ddd, $J=9.6,4.2,3.6 \mathrm{~Hz}, 1 \mathrm{H}), 3.46(\mathrm{ddd}, J=9.0$, 4.8, 4.2 Hz, 1H), $3.36(\mathrm{dd}, J=11.4,9.6 \mathrm{~Hz}, 1 \mathrm{H}), 3.03(\mathrm{ddd}, J=8.4,6.0,5.4 \mathrm{~Hz}, 1 \mathrm{H})$, 2.85 (ddd, $J=8.4,6.0,5.4 \mathrm{~Hz}, 1 \mathrm{H}), 2.11(\mathrm{~s}, 2 \mathrm{H}), 0.89$ (d, $J=4.2 \mathrm{~Hz}, 9 \mathrm{H}), 0.79$ (d, $J=5.4$ $\mathrm{Hz}, 9 \mathrm{H}), 0.77(\mathrm{~d}, J=4.2 \mathrm{~Hz}, 6 \mathrm{H}),-0.004(\mathrm{~s}, 3 \mathrm{H}),-0.02(\mathrm{~d}, J=2.4 \mathrm{~Hz}, 3 \mathrm{H}) ;{ }^{13} \mathrm{C} \mathrm{NMR}$ $\left(150 \mathrm{MHz}, \mathrm{CD}_{3} \mathrm{OD}\right) \delta 174.1,158.5,158.0,138.3,132.0,131.7,131.3,129.5$ (2C), 129.0, $128.7,127.5,118.5,118.1,94.5,92.6,74.8,73.1,68.3,67.7,65.0,64.4,57.2,52.8,37.9$, $33.2,30.8,30.5,30.0,26.67$ (3C), 26.61 (3C), 23.8, 19.4, 14.5, -4.3, -4.6, -4.7, -4.9; CIHRMS Calcd for $\left[\mathrm{C}_{42} \mathrm{H}_{64} \mathrm{~N}_{2} \mathrm{O}_{10} \mathrm{Si}_{2} \mathrm{H}^{+}\right]:$813.4177. Found 813.4177.

$(1 ' S, 4 ' S, 5 ' R, 1 S, 4 S, 5 R)-1-[1 '-N$-carbobenzyloxy-D-tyrosine methoxycarbonyl -5'(tert-butyl-dimethylsilanyloxymethyl)-1',4'-dihydro-5' H-pyran-4'-yloxy] -5-(tertbutyl-dimethylsilanyloxymethyl)-1,4-dihydro-5H-pyran- $N$-4-isovaleric amide (11):

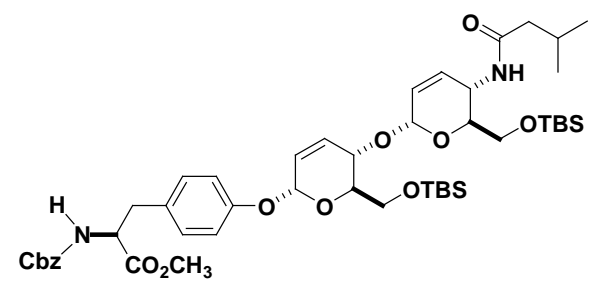


The amine compound 4 (100 mg, $0.12 \mathrm{mmol})$, isovaleric acid (15 mg, $0.15 \mathrm{mmol})$ and DCC (30 mg, $0.14 \mathrm{mmol}$ ) were dissolved in $0.3 \mathrm{~mL}$ of $\mathrm{CH}_{2} \mathrm{Cl}_{2}$ in a round bottom flask and cooled to $0{ }^{\circ} \mathrm{C}$ then DMAP $(2 \mathrm{mg}, 0.01 \mathrm{mmol})$ was added and the reaction mixture was stirred at $0{ }^{\circ} \mathrm{C}$ for 6 hours and on completion, as monitored by TLC, the reaction mixture was diluted with ether and was quenched with $5 \mathrm{~mL}$ of satd. aq. $\mathrm{NaHCO}_{3}$, extracted $\left(3 \times 5\right.$ mL) with $\mathrm{Et}_{2} \mathrm{O}$, dried $\left(\mathrm{Na}_{2} \mathrm{SO}_{4}\right)$, and concentrated under reduced pressure. The crude product was purified using silica gel flash chromatography eluting with EtOAc/hexanes (30:70) to give amide 11 (94 mg, $0.10 \mathrm{mmol}, 85 \%)$ as viscous oil. $R_{f}$ $(30 \%$ EtOAc/hexanes $)=0.70 ;[\alpha]_{\mathrm{D}}^{26}=+4\left(c=1, \mathrm{CH}_{2} \mathrm{Cl}_{2}\right) ; \mathrm{IR}$ (thin film, $\left.\mathrm{cm}^{-1}\right) 2985$, 2927, 1736, 1447, 1372, 1253, 1098, 1043, 938, 846; ${ }^{1} \mathrm{H}$ NMR (600 MHz, $\left.\mathrm{CDCl}_{3}\right) \delta 7.33$ $(\mathrm{m}, 5 \mathrm{H}), 7.00(\mathrm{~m}, 4 \mathrm{H}), 6.20(\mathrm{~d}, J=10.8 \mathrm{~Hz}, 1 \mathrm{H}), 5.92(\mathrm{ddd}, J=10.2,3.0,1.8 \mathrm{~Hz}, 1 \mathrm{H})$, $5.81(\mathrm{~d}, J=10.2 \mathrm{~Hz}, 1 \mathrm{H}), 5.71(\mathrm{ddd}, J=10.2,3.0,2.4 \mathrm{~Hz}, 1 \mathrm{H}), 5.56(\mathrm{~d}, J=2.4 \mathrm{~Hz}, 1 \mathrm{H})$, $5.21(\mathrm{~m}, 1 \mathrm{H}), 5.07(\mathrm{br} \mathrm{s}, 2 \mathrm{H}), 4.58(\mathrm{dd}, J=10.2,5.4 \mathrm{~Hz}, 1 \mathrm{H}), 4.52(\mathrm{~d}, J=8.4 \mathrm{~Hz}, 1 \mathrm{H})$, $4.32(\mathrm{~d}, J=9.0 \mathrm{~Hz}, 1 \mathrm{H}), 3.90(\mathrm{~m}, 1 \mathrm{H}), 3.86(\mathrm{~d}, J=11.4 \mathrm{~Hz}, 1 \mathrm{H}), 3.82(\mathrm{~m}, 2 \mathrm{H}), 3.76$ (ddd, $J=11.4,5.4,4.8 \mathrm{~Hz}, 1 \mathrm{H}), 3.72(\mathrm{dd}, J=11.4,3.0 \mathrm{~Hz}, 1 \mathrm{H}), 3.69$ (s, 3H), 3.66 (dd, $J$ $=3.0,2.4 \mathrm{~Hz}, 1 \mathrm{H}), 3.64(\mathrm{dd}, J=4.2,2.4 \mathrm{~Hz}, 1 \mathrm{H}), 3.03(\mathrm{~m}, 2 \mathrm{H}), 2.11(\mathrm{~m}, 1 \mathrm{H}), 2.00(\mathrm{~d}, J$ $=7.8 \mathrm{~Hz}, 2 \mathrm{H}), 0.93(\mathrm{~d}, J=6.6 \mathrm{~Hz}, 6 \mathrm{H}), 0.87(\mathrm{~s}, 9 \mathrm{H}), 0.80(\mathrm{~s}, 9 \mathrm{H}), 0.04(\mathrm{~s}, 3 \mathrm{H}), 0.03(\mathrm{~s}$, $3 \mathrm{H}), 0.00(\mathrm{~s}, 3 \mathrm{H}),-0.02(\mathrm{~s}, 3 \mathrm{H}) ;{ }^{13} \mathrm{C} \mathrm{NMR}\left(150 \mathrm{MHz}, \mathrm{CDCl}_{3}\right) \delta 171.9,156.6,155.6$, 136.2, 133.1, 130.5, 130.1, 129.0, 128.4 (2C), 128.1 (2C), $128.0(2 \mathrm{C}), 126.1,125.4$ (2C), $117.2(2 \mathrm{C}), 92.8,91.1,71.4,70.0,66.9(2 \mathrm{C}), 66.8,66.7,65.3(2 \mathrm{C}), 62.6,54.8,52.2,37.3$, 27.3, 25.9 (3C), 25.8 (3C), 24.0, 18.3, 18.2, -5.0, -5.1, -5.4, -5.6; CIHRMS Calcd for $\left[\mathrm{C}_{47} \mathrm{H}_{72} \mathrm{~N}_{2} \mathrm{O}_{11} \mathrm{Si}_{2} \mathrm{Na}^{+}\right]$: 919.4572. Found 919.4543. 


\section{1'- $N$-carbobenzyloxy-D-tyrosine methoxycarbonyl -5',5-(tert-butyl-}

\section{dimethylsilanyloxymethyl)-di-1,4- $\alpha$-D-mannose $-N-4$ isovaleric amide (12):}

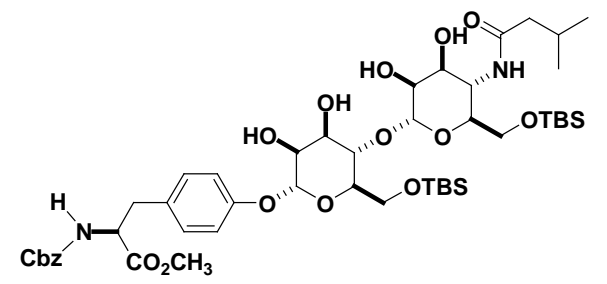

To a $\mathrm{CH}_{2} \mathrm{Cl}_{2}(1.3 \mathrm{~mL})$ solution of diene amide $11(120 \mathrm{mg}, 0.13 \mathrm{mmol})$ at $0{ }^{\circ} \mathrm{C}$ was added a solution of $(50 \% \mathrm{w} / \mathrm{v})$ of $N$-methyl morpholine $N$-oxide / water $(0.1 \mathrm{~mL})$. Crystalline $\mathrm{OsO}_{4}(3.4 \mathrm{mg}, 10 \mathrm{~mol} \%)$ was added and the reaction was stirred for $12 \mathrm{~h}$. The reaction mixture was concentrated and was pipetted directly on to a silica gel column using $\mathrm{CH}_{2} \mathrm{Cl}_{2}(1 \mathrm{~mL})$ in three portions. Impurities were eluted with ether and the product was eluted with $\mathrm{MeOH} / \mathrm{EtOAc} / \mathrm{hexanes}$ (10:40:50). Pure fractions were combined and concentrated to afford bis-manno-amido-tetrol $12(128 \mathrm{mg}, 0.13 \mathrm{mmol}, 80 \%)$ as viscous oil. $R_{f}(90 \%$ EtOAc/MeOH $)=0.40 ;[\alpha]_{\mathrm{D}}^{26}=+12\left(c=1, \mathrm{CH}_{2} \mathrm{Cl}_{2}\right) ;$ IR (thin film, $\left.\mathrm{cm}^{-1}\right)$ 2956, 2928, 1738, 1733, 1510, 1253, 1219, 1123, 986, 835; ${ }^{1} \mathrm{H}$ NMR $(600 \mathrm{MHz}$, $\left.\mathrm{CD}_{3} \mathrm{OD}\right) \delta 7.30(\mathrm{~m}, 5 \mathrm{H}), 6.98(\mathrm{~m}, 4 \mathrm{H}), 5.45(\mathrm{~d}, J=1.2 \mathrm{~Hz}, 1 \mathrm{H}), 5.34(\mathrm{~d}, J=1.8 \mathrm{~Hz}, 1 \mathrm{H})$, $5.22(\mathrm{~d}, J=1.8 \mathrm{~Hz}, 1 \mathrm{H}), 5.01(\mathrm{~d}, J=12.6 \mathrm{~Hz}, 1 \mathrm{H}), 5.00(\mathrm{~d}, J=12.6 \mathrm{~Hz}, 1 \mathrm{H}), 4.50$ (br s, $1 \mathrm{H}), 4.37(\mathrm{dd}, J=8.4,5.4 \mathrm{~Hz}, 1 \mathrm{H}), 4.34(\mathrm{dd}, J=9.0,4.8 \mathrm{~Hz}, 1 \mathrm{H}), 4.06(\mathrm{dd}, J=14.4,7.2$ $\mathrm{Hz}, 1 \mathrm{H}), 4.02(\mathrm{dd}, J=6.6,6.6 \mathrm{~Hz}, 1 \mathrm{H}), 3.99(\mathrm{dd}, J=9.0,4.2 \mathrm{~Hz}, 1 \mathrm{H}), 3.93(\mathrm{dd}, J=6.0$, $4.2 \mathrm{~Hz}, 1 \mathrm{H}), 3.91(\mathrm{~m}, 1 \mathrm{H}), 3.85(\mathrm{~d}, J=11.4 \mathrm{~Hz}, 1 \mathrm{H}), 3.82(\mathrm{dd}, J=6.0,5.4 \mathrm{~Hz}, 1 \mathrm{H}), 3.81$ $(\mathrm{dd}, J=9.6,5.4 \mathrm{~Hz}, 1 \mathrm{H}), 3.73(\mathrm{dd}, J=6.6,3.0 \mathrm{~Hz}, 1 \mathrm{H}), 3.72(\mathrm{~d}, J=3.0 \mathrm{~Hz}, 1 \mathrm{H}), 3.70$ $(\mathrm{dd}, J=3.0,1.8 \mathrm{~Hz}, 1 \mathrm{H}), 3.68(\mathrm{dd}, J=6.0,1.8 \mathrm{~Hz}, 1 \mathrm{H}), 3.65(\mathrm{~s}, 3 \mathrm{H}), 3.60(\mathrm{~m}, 1 \mathrm{H}), 3.03$ $(\mathrm{dd}, J=9.6,4.8 \mathrm{~Hz}, 1 \mathrm{H}), 3.02(\mathrm{dd}, J=9.0,4.8 \mathrm{~Hz}, 1 \mathrm{H}), 2.86(\mathrm{dd}, J=9.0,4.2 \mathrm{~Hz}, 1 \mathrm{H})$, $2.84(\mathrm{dd}, J=9.6,4.2 \mathrm{~Hz}, 1 \mathrm{H}), 2.15(\mathrm{~m}, 1 \mathrm{H}), 2.04(\mathrm{dd}, J=5.4,1.8 \mathrm{~Hz}, 1 \mathrm{H}), 2.02(\mathrm{dd}, J=$ 
4.8, 1.2 Hz, 1H), $0.87(\mathrm{~d}, J=6.0 \mathrm{~Hz}, 6 \mathrm{H}), 0.81(\mathrm{~s}, 9 \mathrm{H}), 0.80(\mathrm{~s}, 9 \mathrm{H}), 0.05(\mathrm{~s}, 3 \mathrm{H}), 0.03$ (s, $3 \mathrm{H}), 0.00(\mathrm{~s}, 3 \mathrm{H}),-0.01(\mathrm{~s}, 3 \mathrm{H}) ;{ }^{13} \mathrm{C} \mathrm{NMR}\left(150 \mathrm{MHz}, \mathrm{CD}_{3} \mathrm{OD}\right) \delta$ 173.0, 172.9, 157.3, 155.9, 137.1, 131.1, 130.3 (2C), 128.4 (2C), 127.9, 127.6 (2C), 116.9 (2C), 101.1, 99.0, 73.7, 73.5, 72.5, 72.1, $71.3(2 \mathrm{C}), 69.7,69.4,66.5,64.1,63.3,56.0,51.6,43.5,36.81$, 30.9, 28.8, 25.8 (3C), 25.5 (3C), 21.8, 18.3, 18.2, -5.7, -5.9, -6.1, -6.3; CIHRMS Calcd for $\left[\mathrm{C}_{47} \mathrm{H}_{76} \mathrm{~N}_{2} \mathrm{O}_{15} \mathrm{Si}_{2} \mathrm{Na}^{+}\right]$: 987.4682. Found 987.4680.

\section{1 '- $N$-carbobenzyloxy-D-tyrosine methoxycarbonyl -5',5-(hydroxy methyl)-di-1,4- $\alpha$ - D-mannose $-N-4$ isovaleric amide (3):}

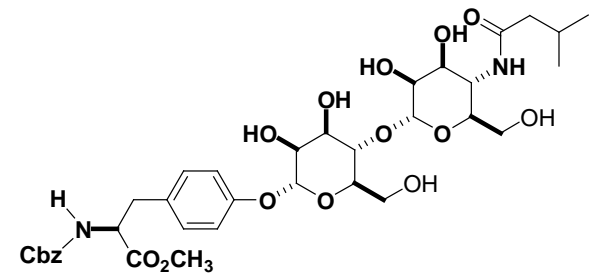

To a THF (0.4 mL, 0.1 M) solution of D-manno-tetrol $12(40 \mathrm{mg}, 0.04 \mathrm{mmol})$ at $0{ }^{\circ} \mathrm{C}$ was added a solution of TBAF in THF $(85 \mu \mathrm{L}, 0.08 \mathrm{mmol})$ and the reaction was stirred for 1 h. The reaction mixture was concentrated and was pipetted directly on to a silica gel column using $\mathrm{CH}_{2} \mathrm{Cl}_{2}(1 \mathrm{~mL})$ in three portions. Impurities were eluted with ether and the product was eluted with $\mathrm{MeOH} / \mathrm{EtOAc/Hexane}$ (20:40:40). Pure fractions were combined and concentrated to afford hexol $3(31 \mathrm{mg}, 0.04 \mathrm{mmol}, 80 \%)$ as viscous oil. $R_{f}$ $(10: 50: 40 \mathrm{MeOH} /$ EtOAc/Hexane $)=0.20 ;[\alpha]_{\mathrm{D}}^{26}=+15\left(c=0.5, \mathrm{CH}_{3} \mathrm{OH}\right) ;$ IR (thin film, $\left.\mathrm{cm}^{-1}\right) 3330,2960,2928,1735,1621,1511,1232,1092,983,829 ;{ }^{1} \mathrm{H}$ NMR $(600 \mathrm{MHz}$, $\left.\mathrm{CD}_{3} \mathrm{OD}\right) \delta 7.30(\mathrm{~m}, 5 \mathrm{H}), 6.99(\mathrm{~m}, 4 \mathrm{H}), 5.39(\mathrm{~d}, J=1.2 \mathrm{~Hz}, 1 \mathrm{H}), 5.33(\mathrm{~d}, J=1.8 \mathrm{~Hz}, 1 \mathrm{H})$, $5.23(\mathrm{~d}, J=1.8 \mathrm{~Hz}, 1 \mathrm{H}), 5.00(\mathrm{~m}, 2 \mathrm{H}), 4.37(\mathrm{dd}, J=5.4,4.8 \mathrm{~Hz}, 1 \mathrm{H}), 4.35(\mathrm{dd}, J=5.4$, $4.2 \mathrm{~Hz}, 1 \mathrm{H}), 4.16(\mathrm{dd}, J=11.4,6.6 \mathrm{~Hz}, 1 \mathrm{H}), 4.01(\mathrm{dd}, J=9.0,3.6 \mathrm{~Hz}, 1 \mathrm{H}), 3.96$ (dd, $J=$ 
9.6, $6.6 \mathrm{~Hz}, 1 \mathrm{H}), 3.91(\mathrm{dd}, J=3.6,1.8 \mathrm{~Hz}, 1 \mathrm{H}), 3.80(\mathrm{dd}, J=9.6,3.0 \mathrm{~Hz}, 1 \mathrm{H}), 3.74(\mathrm{dd}, J$ $=8.4,4.8 \mathrm{~Hz}, 1 \mathrm{H}), 3.71(\mathrm{dd}, J=8.4,4.2 \mathrm{~Hz}, 1 \mathrm{H}), 3.67(\mathrm{~d}, J=3.0 \mathrm{~Hz}, 1 \mathrm{H}), 3.65(\mathrm{~m}, 3 \mathrm{H})$, $3.60(\mathrm{dd}, J=9.0,4.2 \mathrm{~Hz}, 1 \mathrm{H}), 3.58(\mathrm{dd}, J=9.6,4.2 \mathrm{~Hz}, 1 \mathrm{H}), 3.53(\mathrm{dd}, J=5.4,3.0 \mathrm{~Hz}$, 1H), $3.15(\mathrm{dd}, J=5.4,2.4 \mathrm{~Hz}, 1 \mathrm{H}), 3.05(\mathrm{dd}, J=9.0,4.8 \mathrm{~Hz}, 1 \mathrm{H}), 3.04(\mathrm{dd}, J=9.6,4.8$ Hz, 1H), $2.84(\mathrm{~m}, 2 \mathrm{H}), 2.22(\mathrm{dd}, J=4.8,1.8 \mathrm{~Hz}, 1 \mathrm{H}), 2.19(\mathrm{dd}, J=5.4,1.8 \mathrm{~Hz}, 1 \mathrm{H}), 2.04$ (m, 1H), $0.92(\mathrm{~d}, J=6.6 \mathrm{~Hz}, 6 \mathrm{H}) ; \mathrm{NMR}\left(150 \mathrm{MHz}, \mathrm{CD}_{3} \mathrm{OD}\right) \delta 174.5,174.0,158.4$, 156.8, 138.2, 132.2, 131.4 (2C), 129.5 (2C), 129.0, 128.7 (2C), 117.8 (2C), 102.8, 100.1, 75.7, 74.9, 74.0, 73.8, 73.6, 73.0, 72.5, 70.6, 67.6, 65.2, 62.8, 62.4, 57.1, 52.8, 44.4, 37.9, 26.8, 22.9; CIHRMS Calcd for $\left[\mathrm{C}_{35} \mathrm{H}_{48} \mathrm{~N}_{2} \mathrm{O}_{15} \mathrm{Na}^{+}\right]$: 759.2947. Found 759.2940.

\section{1'-N-carbobenzyloxy-D-tyrosine methoxycarbonyl -5',5-(tert-butyl- dimethylsilanyloxymethyl)-2,3,2',3'-di acetonide-bis-1,4- $\alpha$-D-mannose- $N$-4- isovaleric amide (13) :}

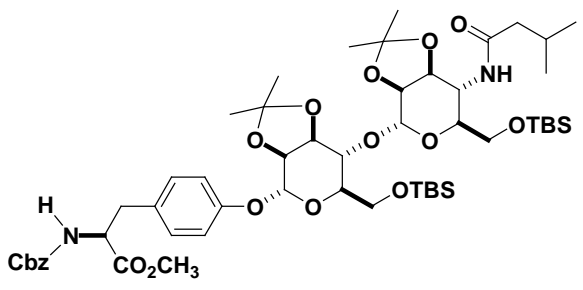

To a $\mathrm{CH}_{2} \mathrm{Cl}_{2}(0.1 \mathrm{~mL}, 1.0 \mathrm{M})$ solution of D-manno-amido-tetrol $12(10 \mathrm{mg}, 0.01 \mathrm{mmol})$ and 2,2-dimethoxypropane $(2.4 \mathrm{mg}, 0.02 \mathrm{mmol})$ at $0{ }^{\circ} \mathrm{C}$ was added CSA $(0.23 \mathrm{mg}, 10$ mol\%) and the reaction was stirred for $3 \mathrm{~h}$. The reaction mixture was concentrated and was pipetted directly on to a silica gel column using $\mathrm{CH}_{2} \mathrm{Cl}_{2}(1 \mathrm{~mL})$ in three portions. Impurities were eluted with ether and the product was eluted with EtOAc/Hexane (50:50). Pure fractions were combined and concentrated to afford di-acetonide $\mathbf{1 3}$ (9 mg, $0.01 \mathrm{mmol}, 80 \%)$ as viscous oil. $R_{f}(50 \%$ EtOAc/Hexane $)=0.40 ;[\alpha]_{\mathrm{D}}^{26}=+60(c=1$, 
$\mathrm{CH}_{2} \mathrm{Cl}_{2}$ ); IR (thin film, $\mathrm{cm}^{-1}$ ) 2951, 2930, 1731, 1498, 1228, 1009, 831; ${ }^{1} \mathrm{H}$ NMR (600 $\left.\mathrm{MHz}, \mathrm{CDCl}_{3}\right) \delta 7.34(\mathrm{~m}, 5 \mathrm{H}), 6.98(\mathrm{~m}, 4 \mathrm{H}), 5.77(\mathrm{~d}, J=10.8 \mathrm{~Hz}, 1 \mathrm{H}), 5.39(\mathrm{~d}, J=8.4$ $\mathrm{Hz}, 1 \mathrm{H}), 5.20(\mathrm{~d}, J=7.8 \mathrm{~Hz}, 1 \mathrm{H}), 5.09(\mathrm{br} \mathrm{s}, 2 \mathrm{H}), 4.62(\mathrm{dd}, J=7.2,4.8 \mathrm{~Hz}, 1 \mathrm{H}), 4.44$ $(\mathrm{dd}, J=6.0,1.2 \mathrm{~Hz}, 1 \mathrm{H}), 4.32(\mathrm{~d}, J=5.4 \mathrm{~Hz}, 1 \mathrm{H}), 4.16(\mathrm{~d}, J=5.4 \mathrm{~Hz}, 1 \mathrm{H}), 4.09(\mathrm{~d}, J=$ $4.8 \mathrm{~Hz}, 1 \mathrm{H}), 4.08(\mathrm{dd}, J=10.2,4.8 \mathrm{~Hz}, 1 \mathrm{H}), 3.95(\mathrm{~m}, \mathrm{H}), 3.72(\mathrm{~s}, 3 \mathrm{H}), 3.69(\mathrm{~m}, 2 \mathrm{H}), 3.69$ (m, 1H), $3.62(\mathrm{~d}, J=3.6 \mathrm{~Hz}, 1 \mathrm{H}), 3.55(\mathrm{dd}, J=10.2,3.0 \mathrm{~Hz}, 1 \mathrm{H}), 3.09(\mathrm{dd}, J=13.2,6.0$ $\mathrm{Hz}, 1 \mathrm{H}), 3.03(\mathrm{dd}, J=12.8,6.0 \mathrm{~Hz}, 1 \mathrm{H}), 2.16(\mathrm{~s}, 1 \mathrm{H}), 2.15(\mathrm{~m}, 1 \mathrm{H}), 2.12(\mathrm{dd}, J=4.8,1.2$ Hz, 1H), $2.09(\mathrm{~m}, 1 \mathrm{H}), 2.05(\mathrm{~d}, J=3.6 \mathrm{~Hz}, 1 \mathrm{H}), 1.65(\mathrm{~s}, 3 \mathrm{H}), 1.50(\mathrm{~s}, 3 \mathrm{H}), 1.38(\mathrm{~s}, 3 \mathrm{H})$, 1.35 (s, 3H), 0.95 (d, J = 6.6 Hz, 6H), 0.87 (s, 9H), 0.84 (s, 9H), 0.42 (s, 3H), 0.03 (s, 6H), 0.02 (s, 3H); ${ }^{13} \mathrm{C}$ NMR (150 MHz, $\left.\mathrm{CDCl}_{3}\right) \delta 171.9,156.6,155.6,143.8,136.2$, 133.3, 130.2, 130.1 (2C), 129.1 (2C), $128.6(2 \mathrm{C}), 128.5,128.1,128.0$ (2C), 128.8, 126.6, 117.0 (2C), 92.9 (2C), 89.8 (2C), 76.7 (2C), 71.2 (2C), 66.9, 66.5, 62.9 (2C), 62.5, 54.8 (2C), 52.2, $37.3(2 \mathrm{C}), 25.85$ (3C), 25.83 (3C), 25.7 (2C), 18.35, 18.32, -5.1, -5.3 (2C), 5.4; CIHRMS Calcd for $\left[\mathrm{C}_{53} \mathrm{H}_{84} \mathrm{~N}_{2} \mathrm{O}_{15} \mathrm{Si}_{2} \mathrm{H}^{+}\right]$: 1045.5488. Found 1045.5503.

(1'S,4'S,5'R,1S,4S,5R)-1-[1'- $N$-carbobenzyloxy-D-tyrosine methoxycarbonyl -5'-(tertbutyl-dimethylsilanyloxymethyl)-tetrahydro-pyran-4'-yloxy]-5-(tert-butyldimethylsilanyloxymethyl) -tetrahydro-pyran- $N$-4-isovaleric amide (14):

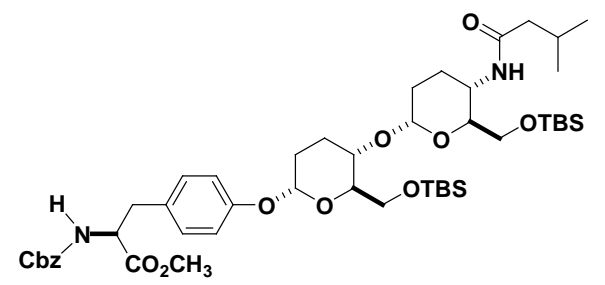

The dieno-amide compound 11 (70 mg, $0.07 \mathrm{mmol})$ and $o-\mathrm{NO}_{2} \mathrm{C}_{6} \mathrm{H}_{4} \mathrm{SO}_{2} \mathrm{NHNH}_{2}(170$ $\mathrm{mg}, 1.17 \mathrm{mmol}$ ) were dissolved in $0.8 \mathrm{~mL}$ of $\mathrm{CH}_{2} \mathrm{Cl}_{2}$ in a round bottom flask and cooled 
$0{ }^{\circ} \mathrm{C}$ under nitrogen atmosphere then triethylamine $(112 \mathrm{mg}, 1.56 \mathrm{mmol})$ was added and the reaction mixture was stirred at $0{ }^{\circ} \mathrm{C}$ for 12 hours and on completion, as monitored by TLC. The reaction mixture was concentrated and was pipetted directly on to a silica gel column using $\mathrm{CH}_{2} \mathrm{Cl}_{2}(1 \mathrm{~mL})$ in three portions. The crude product was purified using silica gel flash chromatography eluting with EtOAc/hexanes (25:75) to give di-deoxy pyranoside $14(68 \mathrm{mg}, 0.07 \mathrm{mmol}, 95 \%)$ as viscous oil. $R_{f}(30 \% \mathrm{EtOAc} / \mathrm{Hexane})=0.50$; $[\alpha]_{\mathrm{D}}^{26}=+37\left(c 1, \mathrm{CH}_{2} \mathrm{Cl}_{2}\right) ; \mathrm{IR}\left(\right.$ thin film, $\left.\mathrm{cm}^{-1}\right)$ 2932, 2927, 1725, 1509, 1220, 1055, 965, 836; ${ }^{1} \mathrm{H}$ NMR (600 MHz, $\left.\mathrm{CDCl}_{3}\right) \delta 7.33(\mathrm{~m}, 5 \mathrm{H}), 6.98(\mathrm{~m}, 4 \mathrm{H}), 5.43(\mathrm{br} \mathrm{s}, 1 \mathrm{H}), 5.26(\mathrm{~d}$, $J=7.8 \mathrm{~Hz}, 1 \mathrm{H}), 5.07(\mathrm{br} \mathrm{s}, 2 \mathrm{H}), 4.58(\mathrm{dd}, J=12.8,5.4 \mathrm{~Hz}, 1 \mathrm{H}), 3.87(\mathrm{dd}, J=9.0,7.2 \mathrm{~Hz}$, $1 \mathrm{H}), 3.73(\mathrm{~d}, J=3.0 \mathrm{~Hz}, 1 \mathrm{H}), 3.72(\mathrm{~m}, 2 \mathrm{H}), 3.69(\mathrm{~m}, 3 \mathrm{H}), 3.65(\mathrm{dd}, J=6.0,5.4 \mathrm{~Hz}, 1 \mathrm{H})$, $3.63(\mathrm{dd}, J=6.0,4.8 \mathrm{~Hz}, 1 \mathrm{H}), 3.50(\mathrm{dd}, J=10.2,4.2 \mathrm{~Hz}, 1 \mathrm{H}), 3.47(\mathrm{~m}, 1 \mathrm{H}), 3.03(\mathrm{dd}, J=$ 14.4, $5.4 \mathrm{~Hz}, 1 \mathrm{H}), 3.01(\mathrm{dd}, J=14.4,5.4 \mathrm{~Hz}, 1 \mathrm{H}), 2.13(\mathrm{~m}, 1 \mathrm{H}), 2.01(\mathrm{dd}, J=13.2,7.8$ $\mathrm{Hz}, 1 \mathrm{H}), 1.99(\mathrm{dd}, J=13.2,7.8 \mathrm{~Hz}, 1 \mathrm{H}), 1.95(\mathrm{~m}, 1 \mathrm{H}), 1.85(\mathrm{dd}, J=8.4,4.2 \mathrm{~Hz}, 1 \mathrm{H})$, $1.82(\mathrm{~m}, 1 \mathrm{H}), 1.79(\mathrm{dd}, J=8.4,3.6 \mathrm{~Hz}, 1 \mathrm{H}), 1.76(\mathrm{dd}, J=7.8,3.6 \mathrm{~Hz}, 1 \mathrm{H}), 1.74(\mathrm{dd}, J=$ 7.8, 4.2 Hz, 1H), $1.68(\mathrm{~m}, 2 \mathrm{H}), 1.06(\mathrm{~m}, 2 \mathrm{H}), 0.88(\mathrm{~d}, J=6.0 \mathrm{~Hz}, 6 \mathrm{H}), 0.81(\mathrm{~s}, 9 \mathrm{H}), 0.80$ (s, 9H), 0.06 (s, 3H), 0.03 (s, 3H), -0.02 (s, 3H), -0.03 (s, 3H); ${ }^{13} \mathrm{C}$ NMR (150 MHz, $\left.\mathrm{CDCl}_{3}\right) \delta 172.0,156.2,155.7,136.2,130.0(2 \mathrm{C}), 128.6,128.4$ (2C), 128.1 (2C), 128.0 (2C), 116.9 (2C), 94.9, 90.8, 74.0, 73.5, 72.1, 67.2, 66.9, 64.3, 63.5, 54.8, 54.7, 52.2 (2C), 47.3, 47.2, 37.3, 29.4, 28.7, 26.0 (3C), 25.9 (3C), 25.8, 22.2, 18.36, 18.33, -5.0, -5.1, 5.2, -5.3; CIHRMS Calcd for $\left[\mathrm{C}_{47} \mathrm{H}_{76} \mathrm{~N}_{2} \mathrm{O}_{11} \mathrm{Si}_{2} \mathrm{Na}^{+}\right]$: 923.4885. Found 923.4886. 


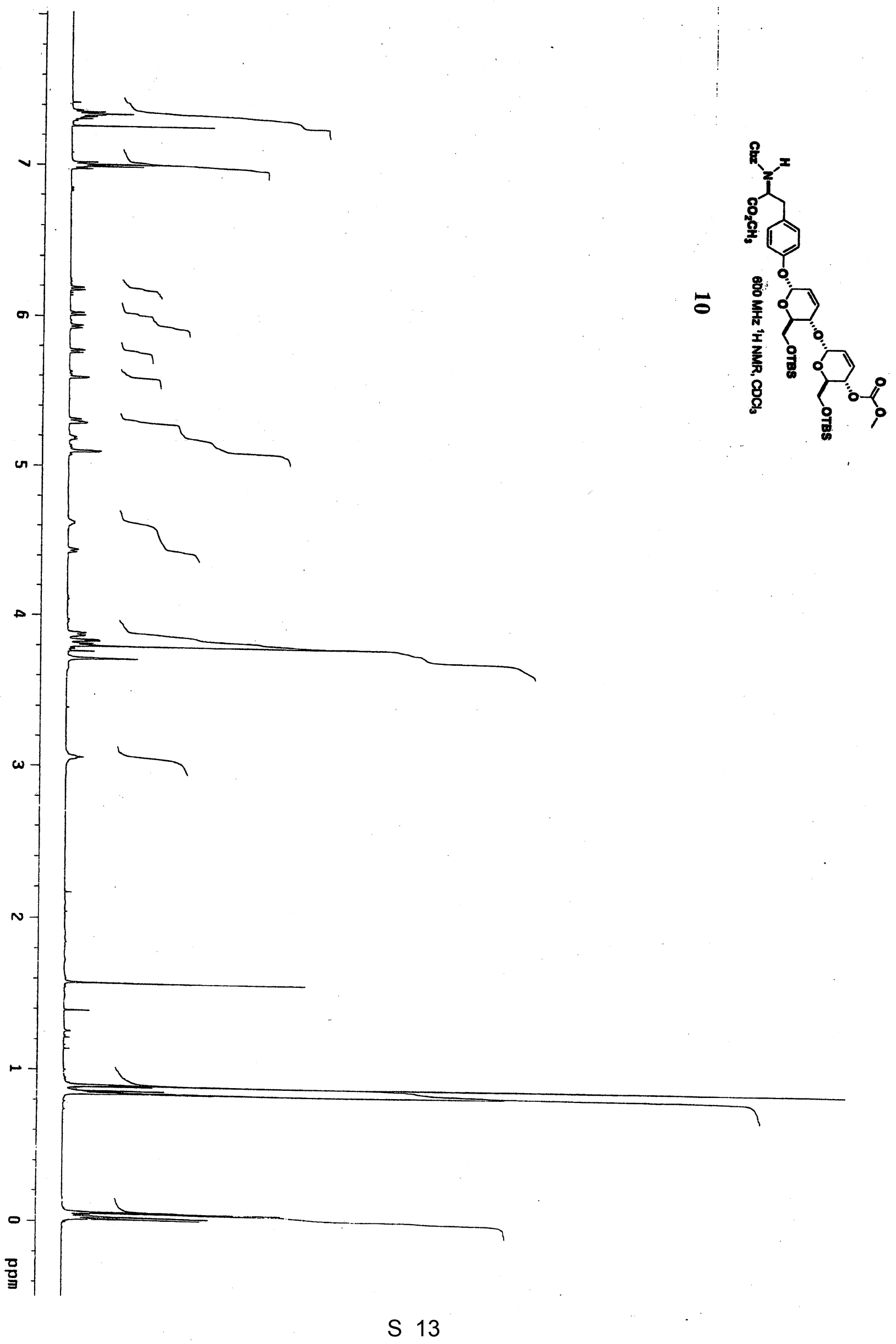




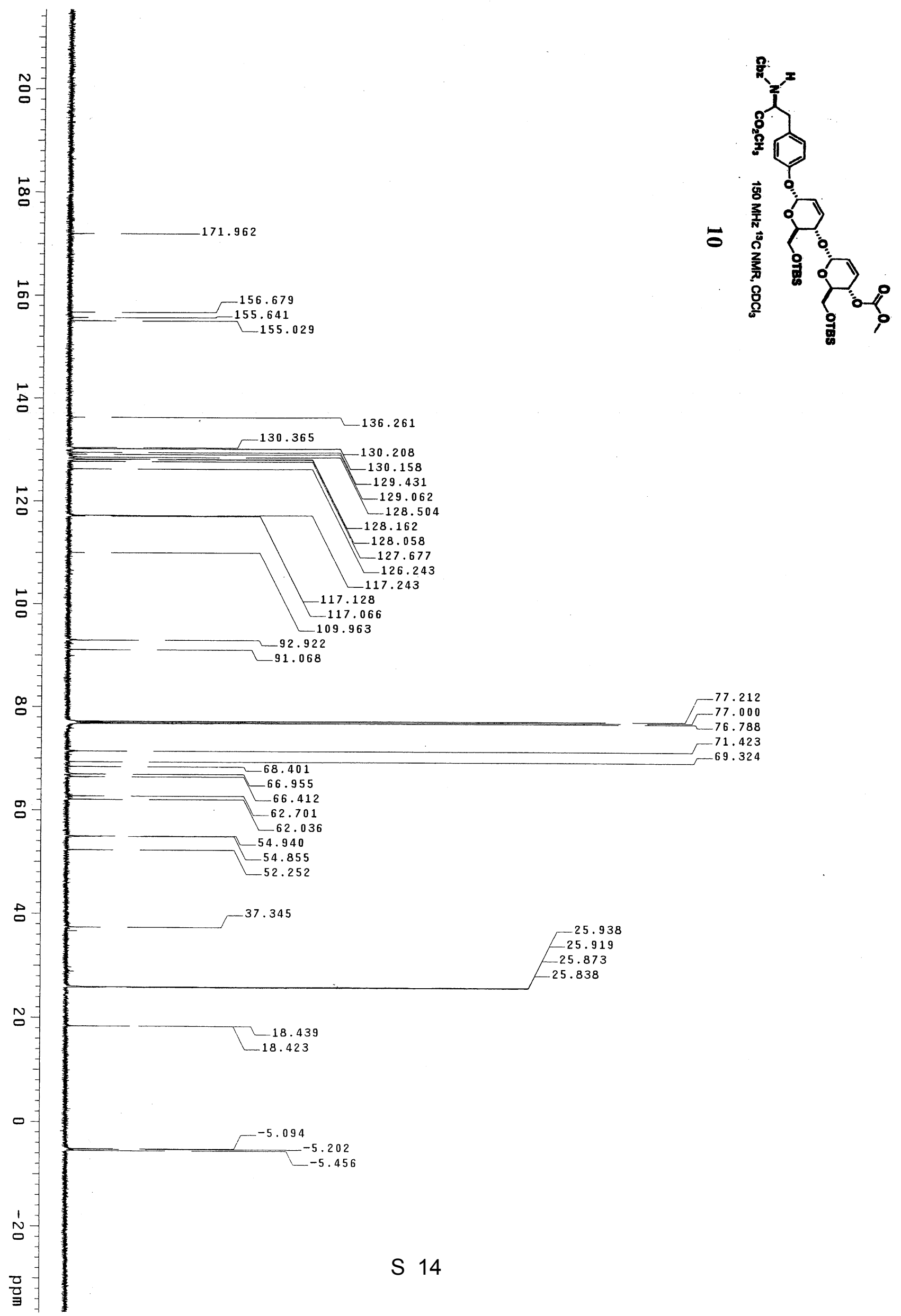




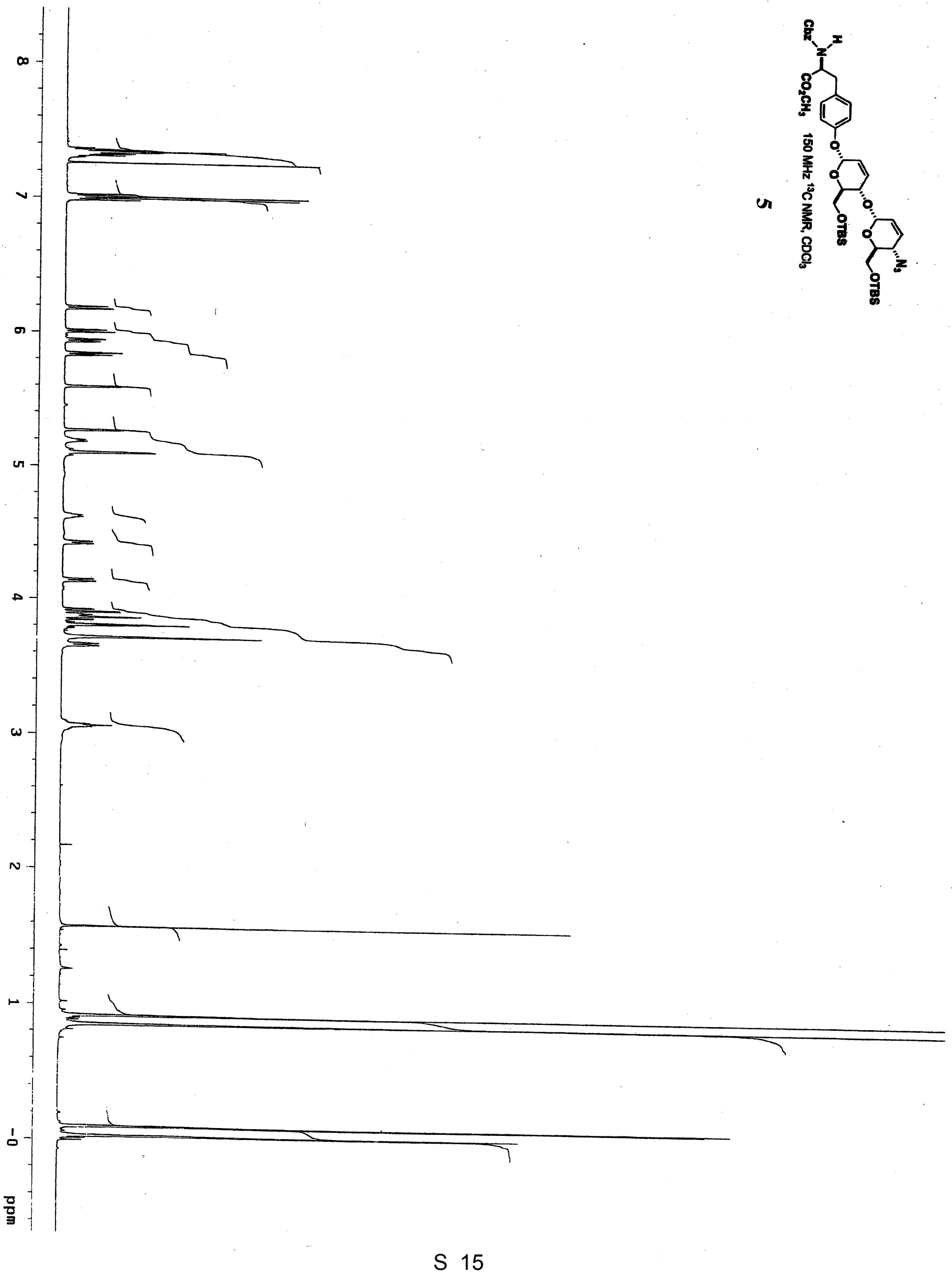




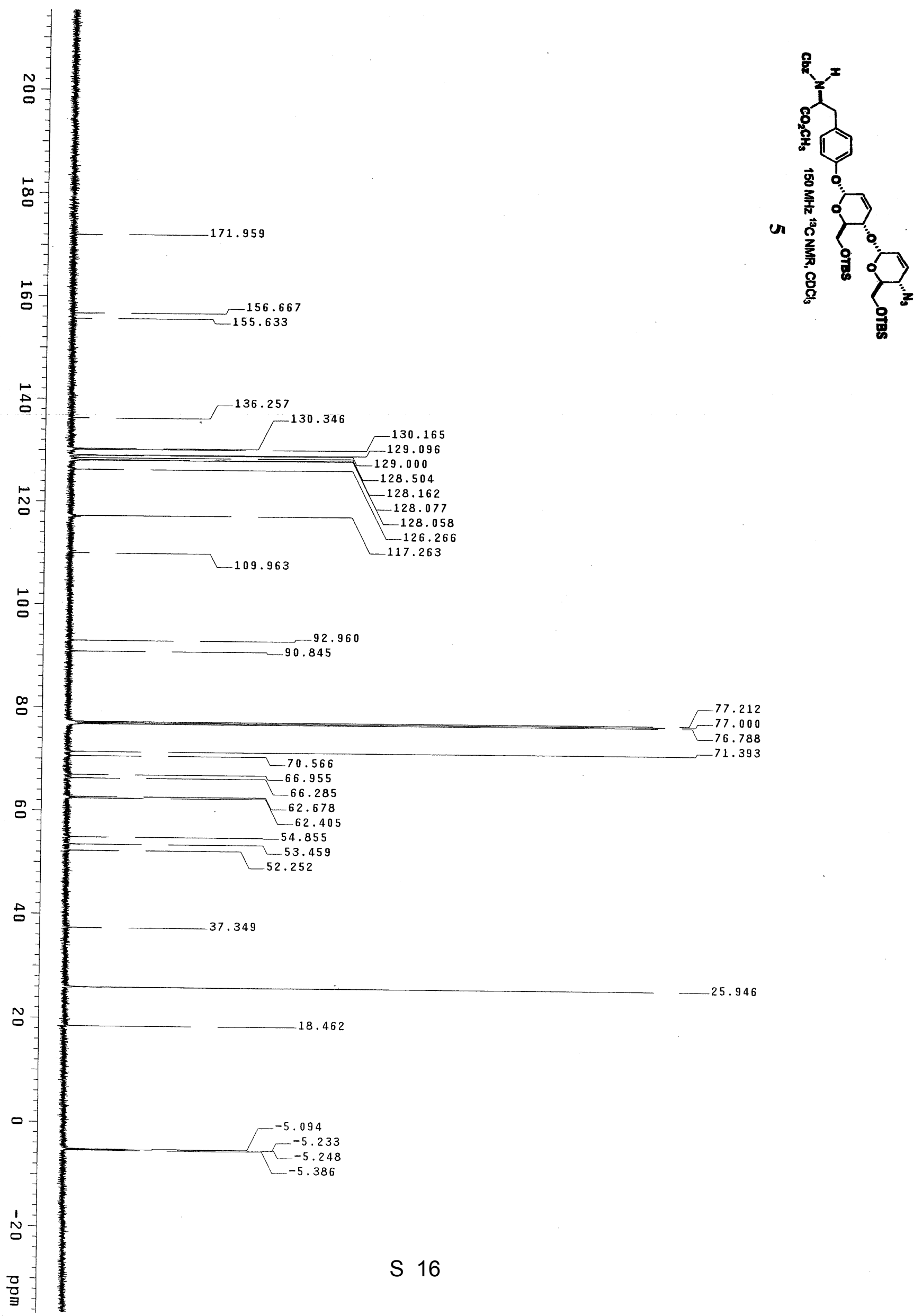




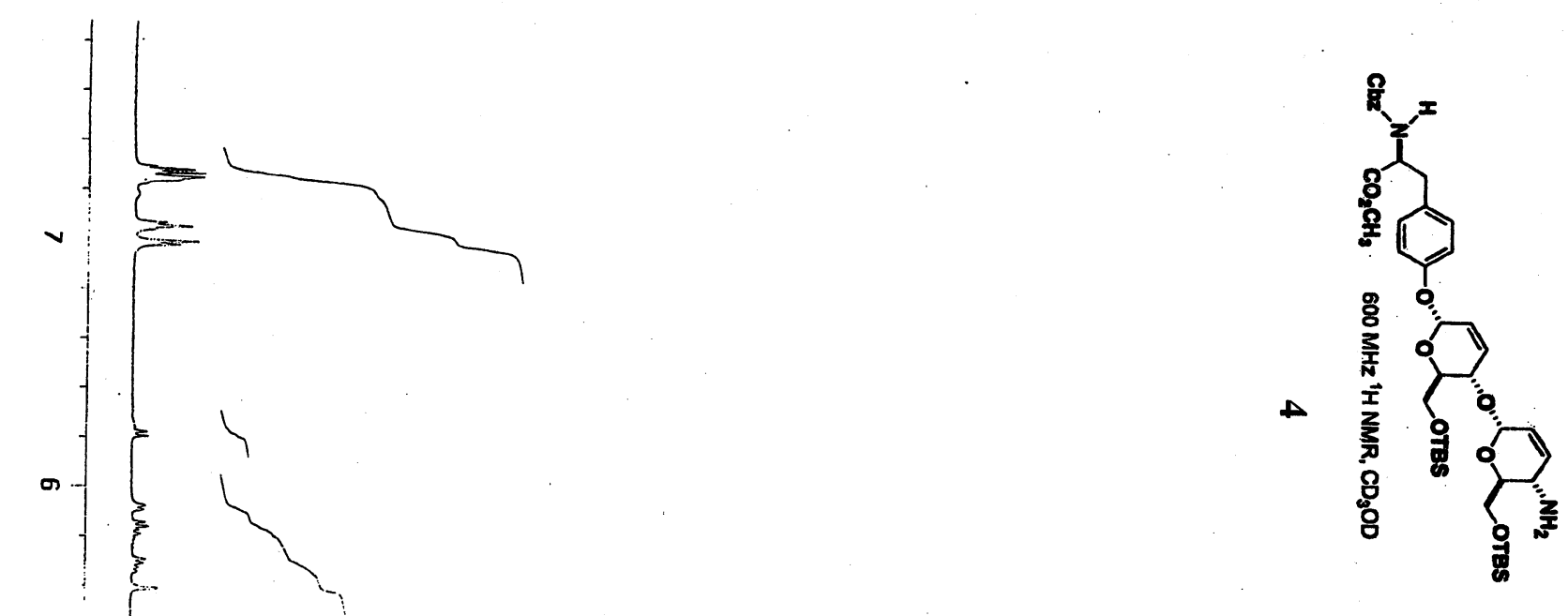




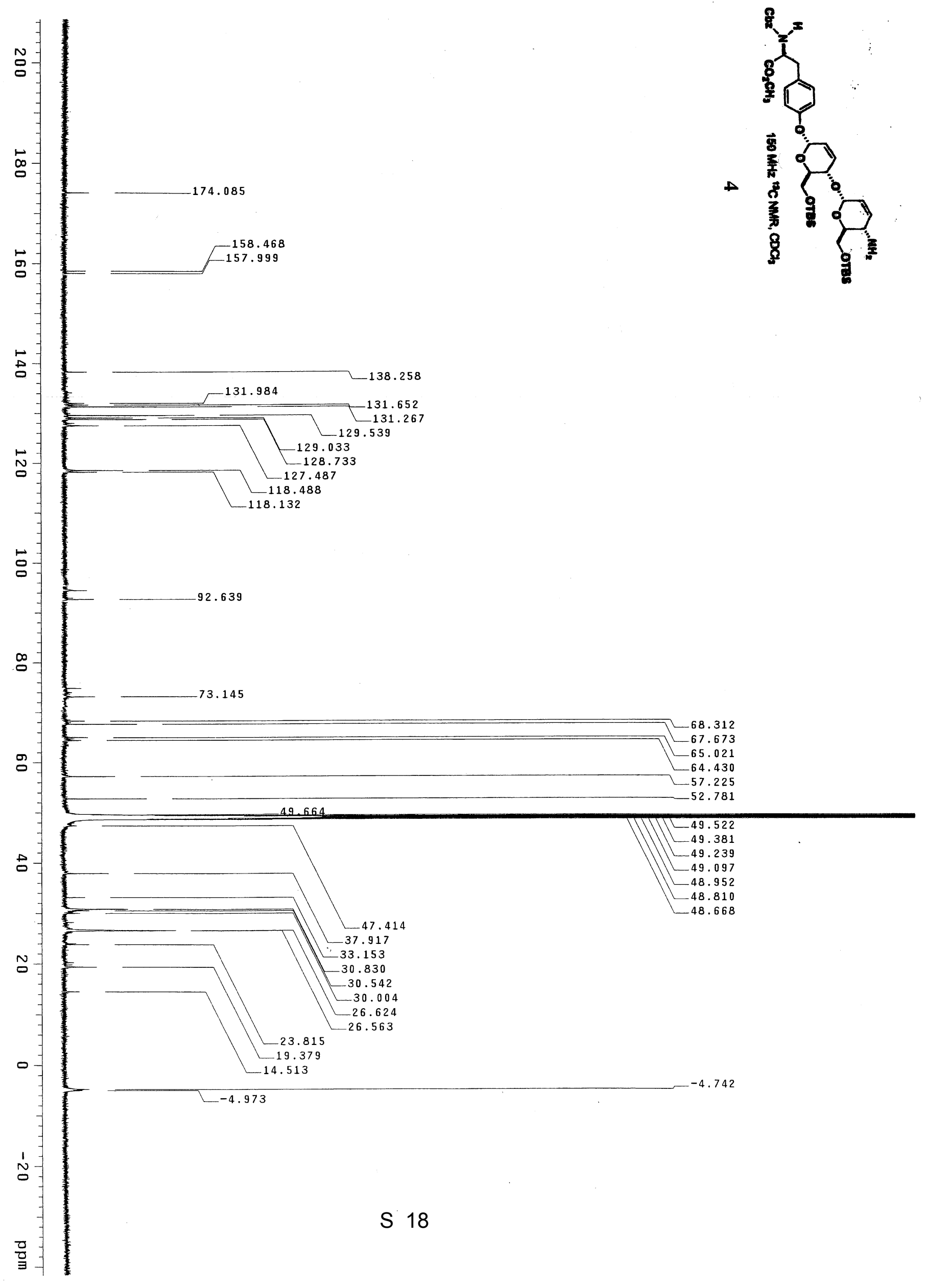




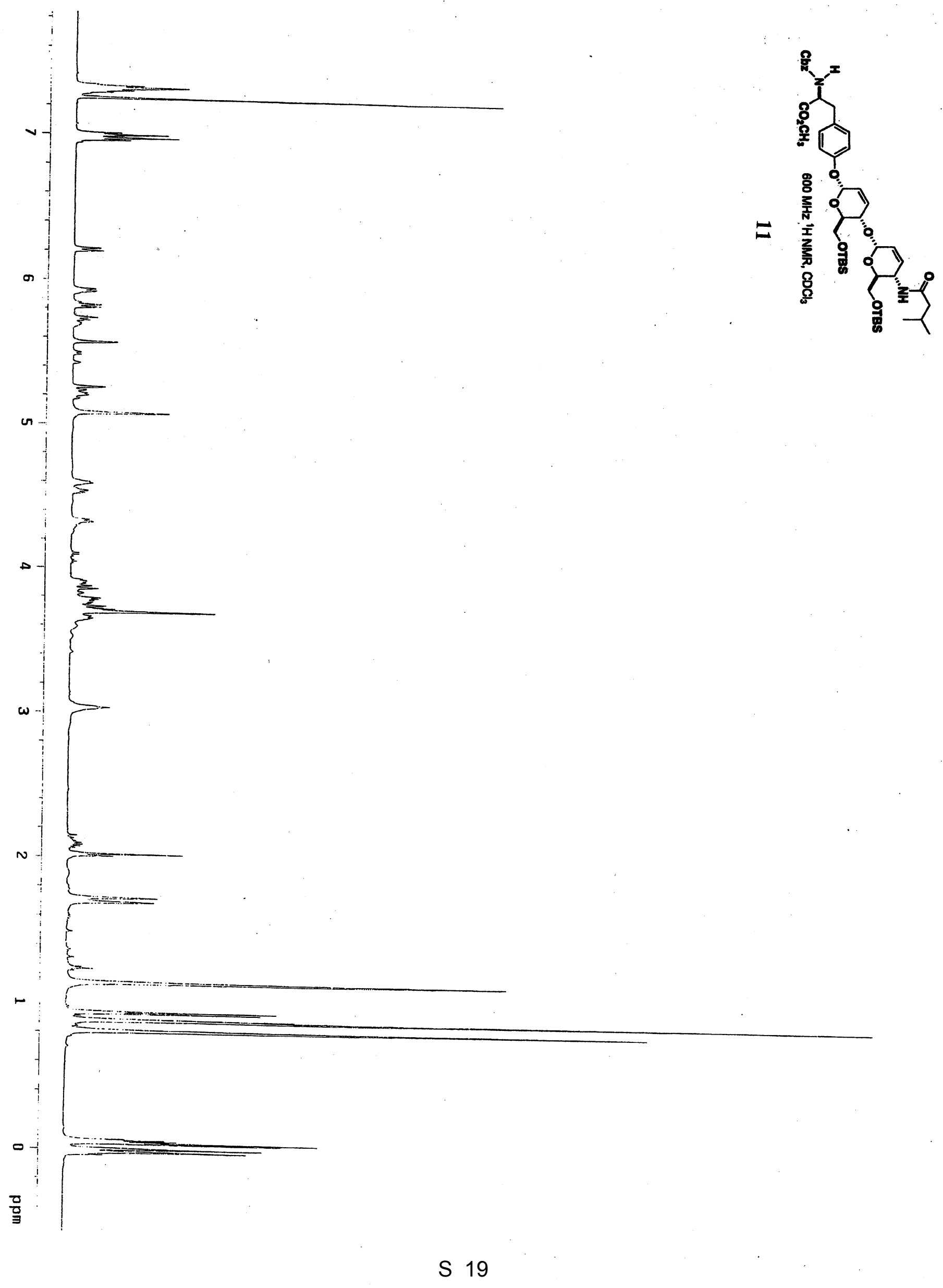




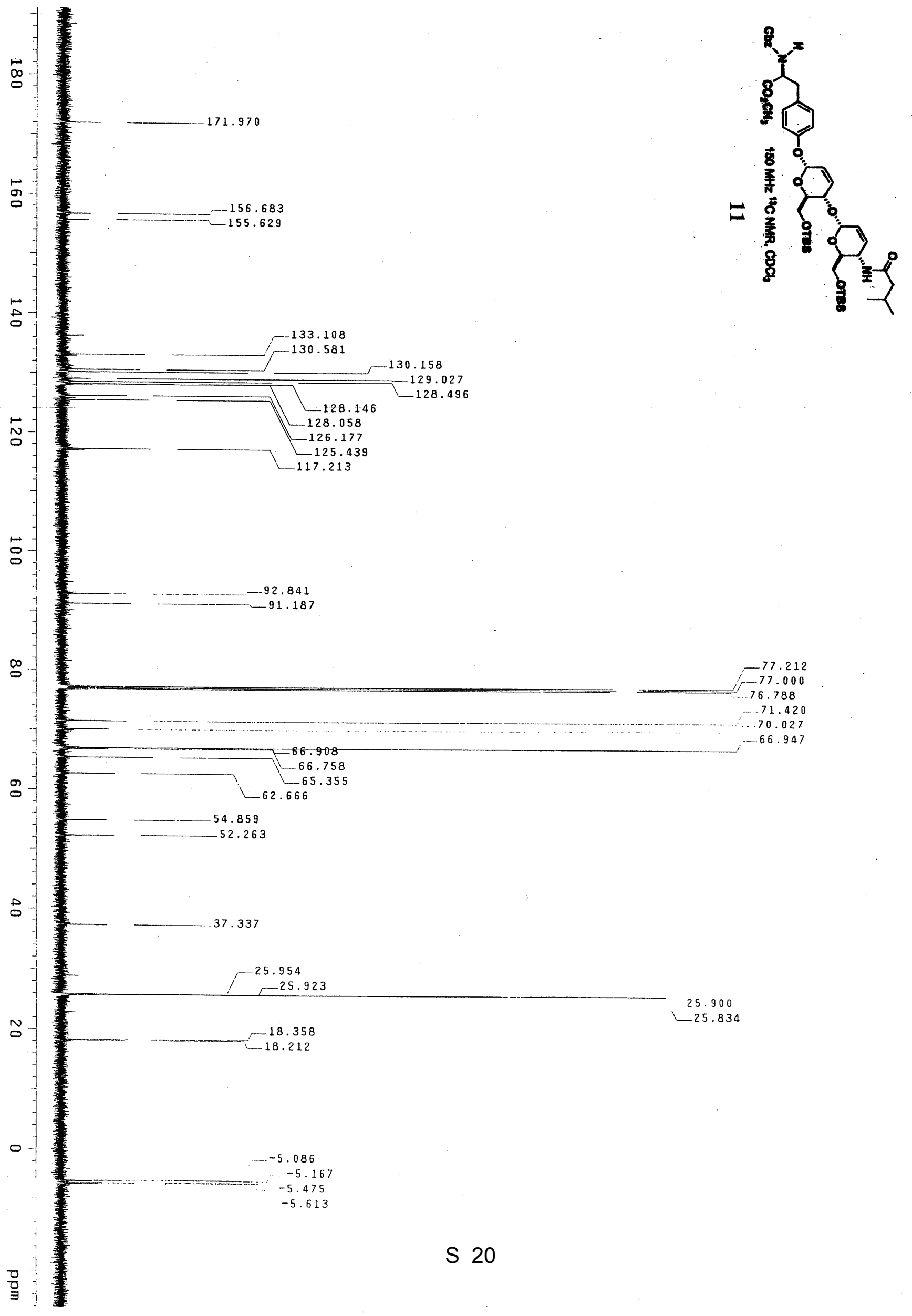




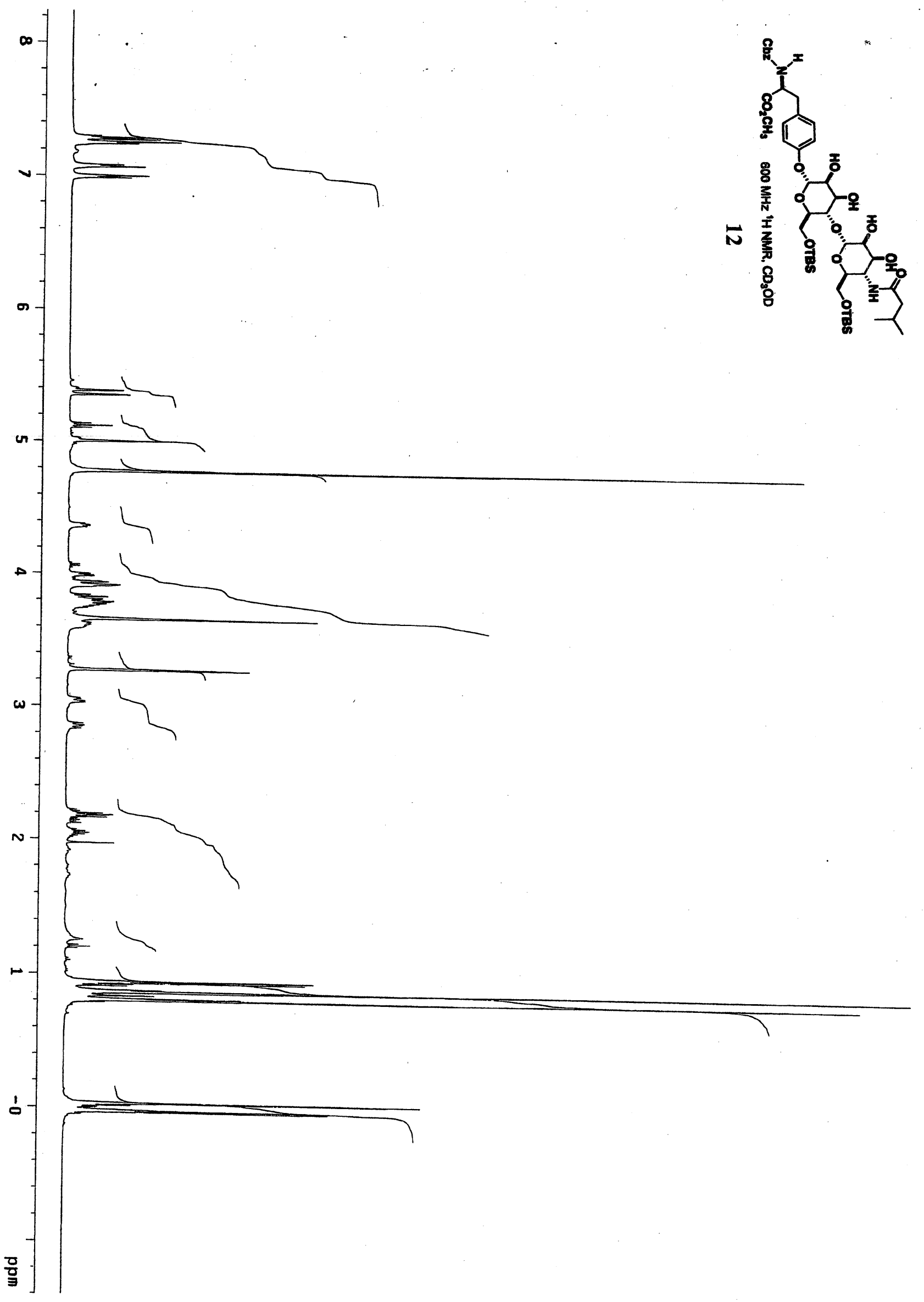




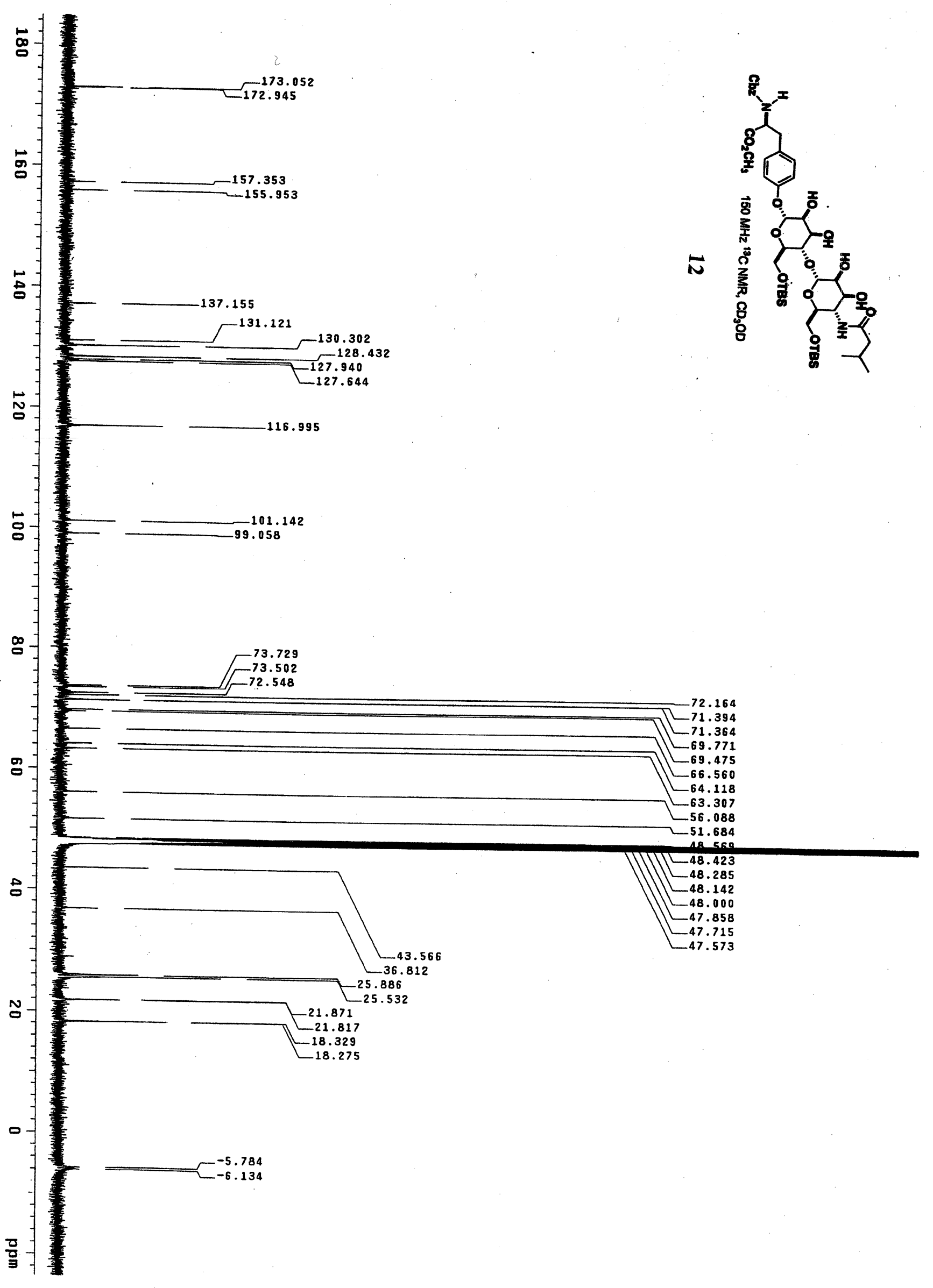




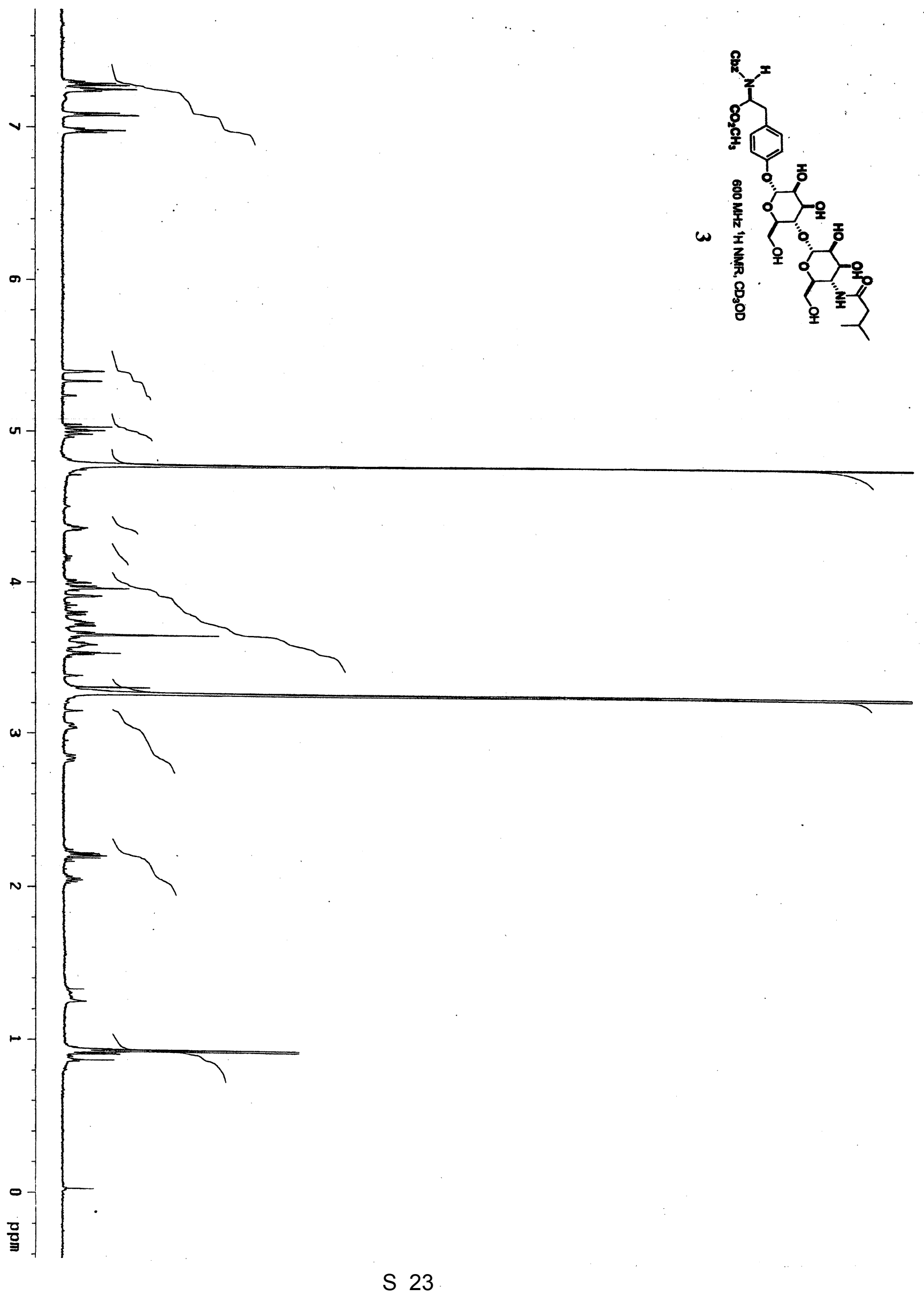




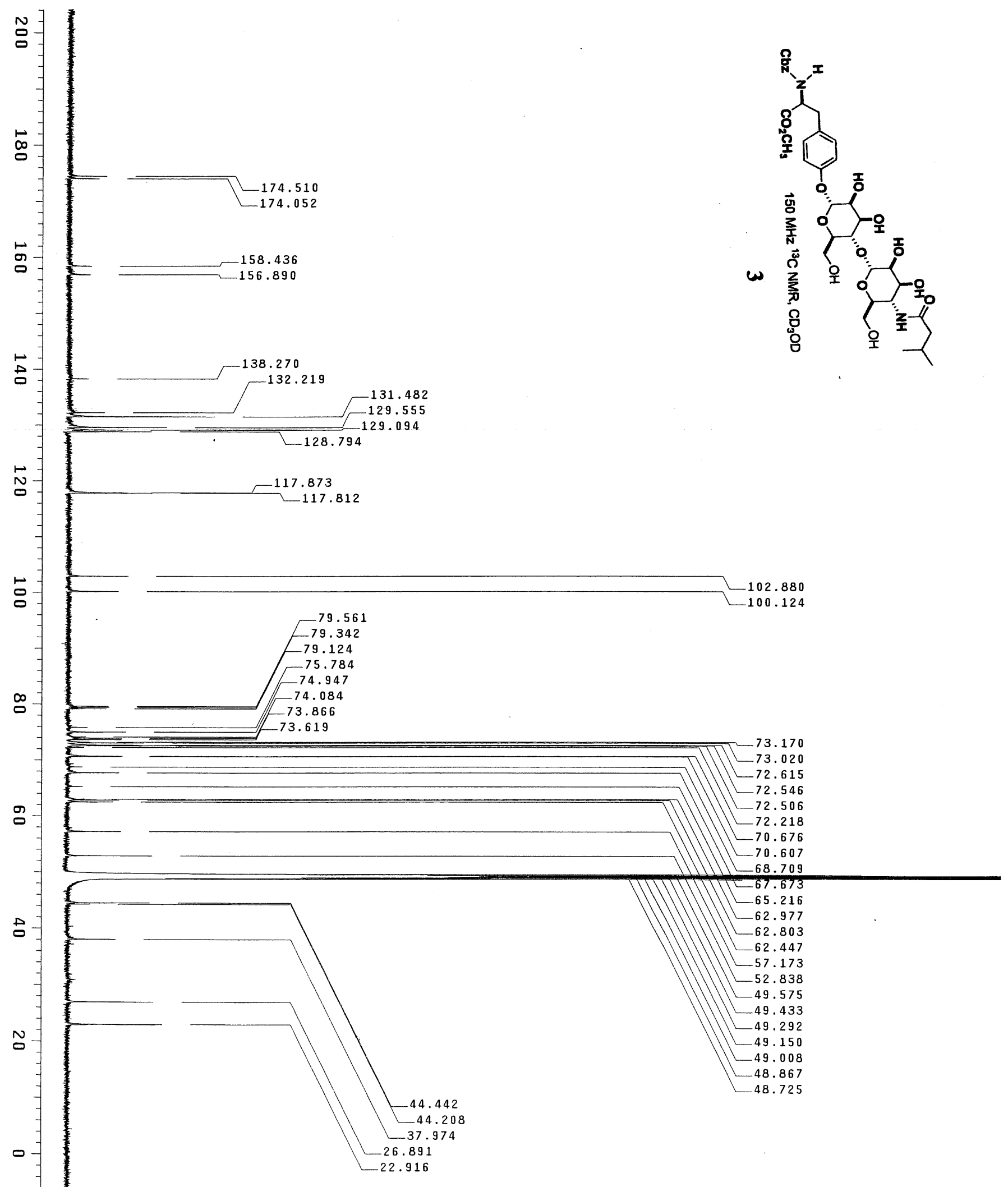




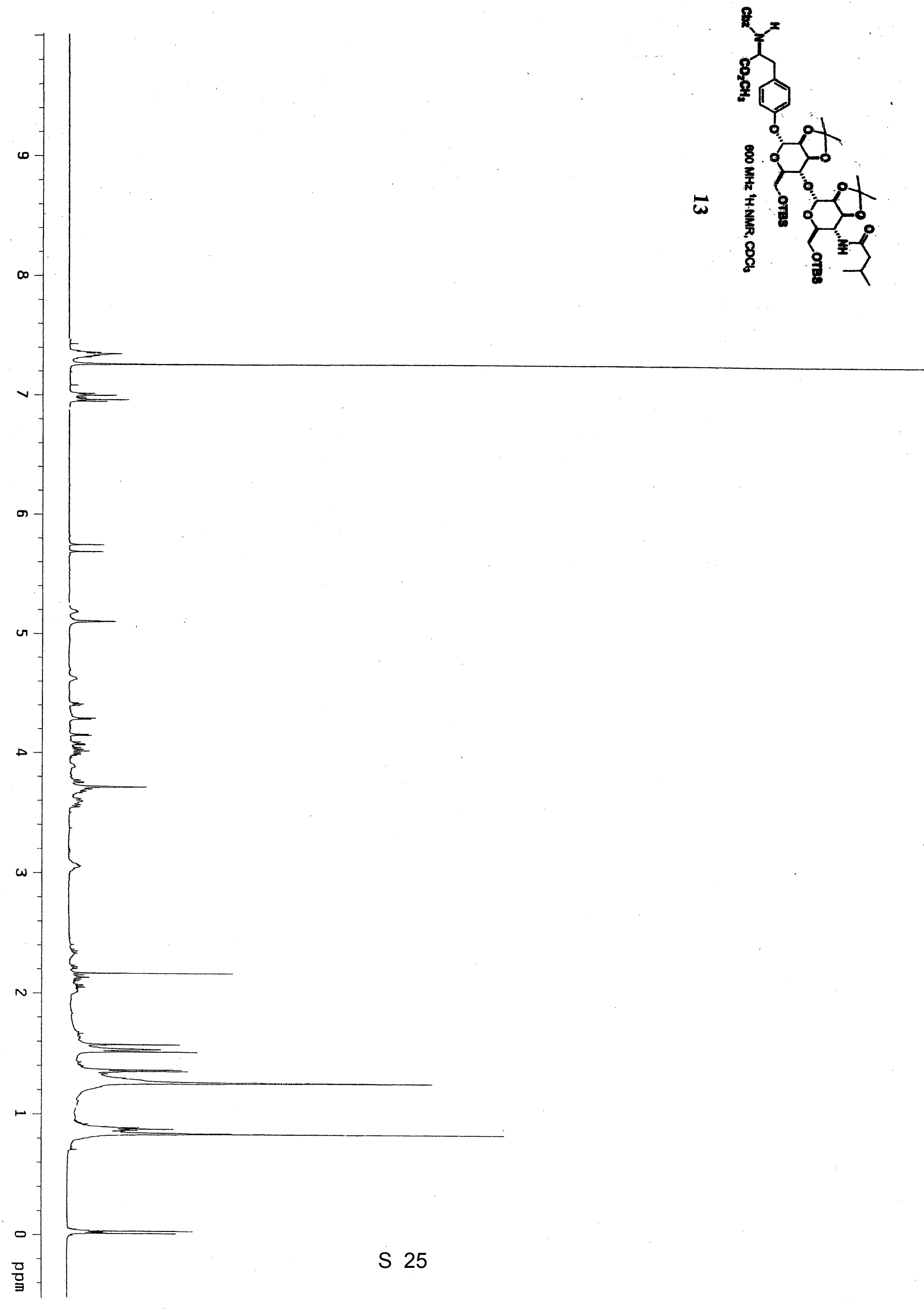



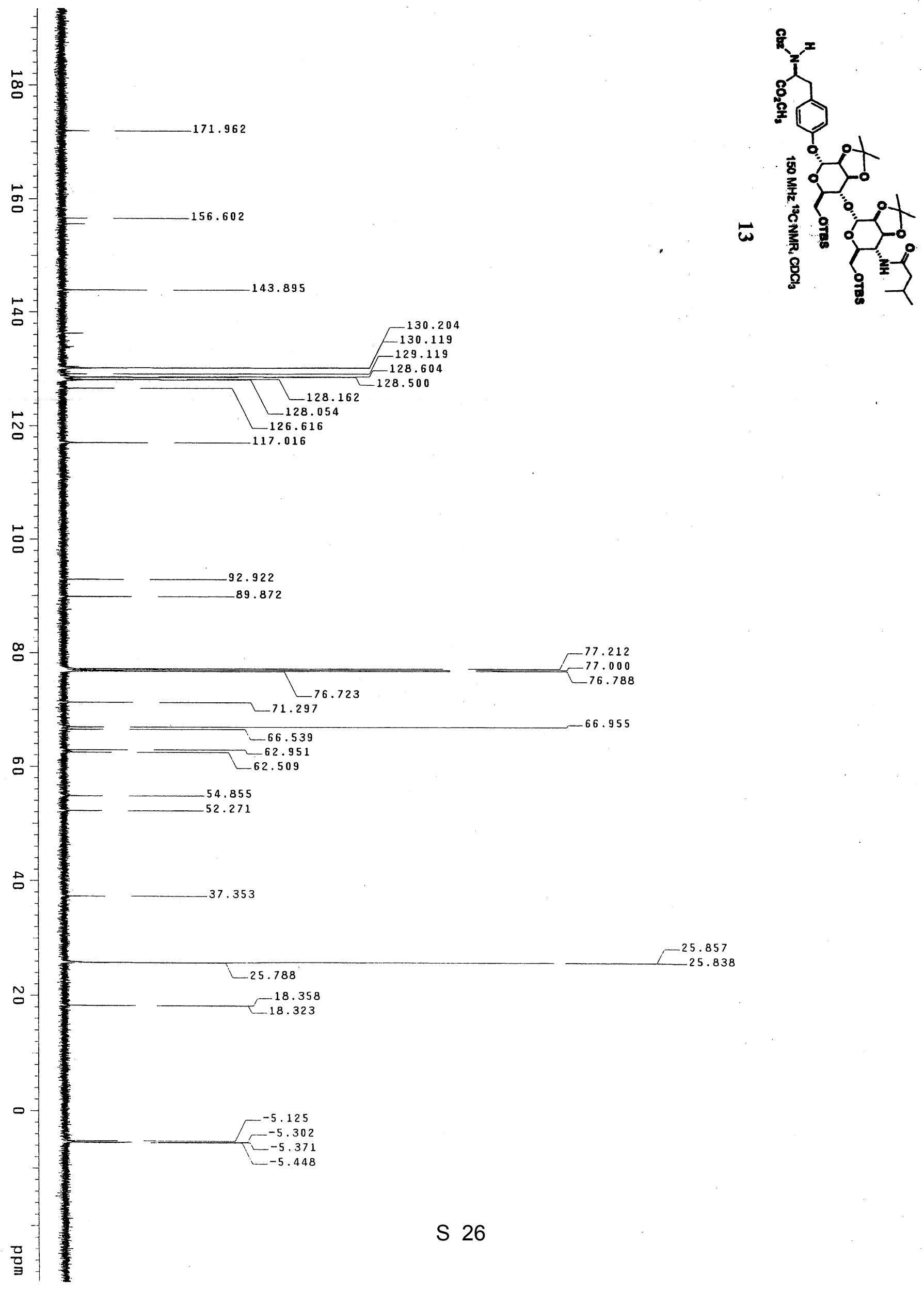


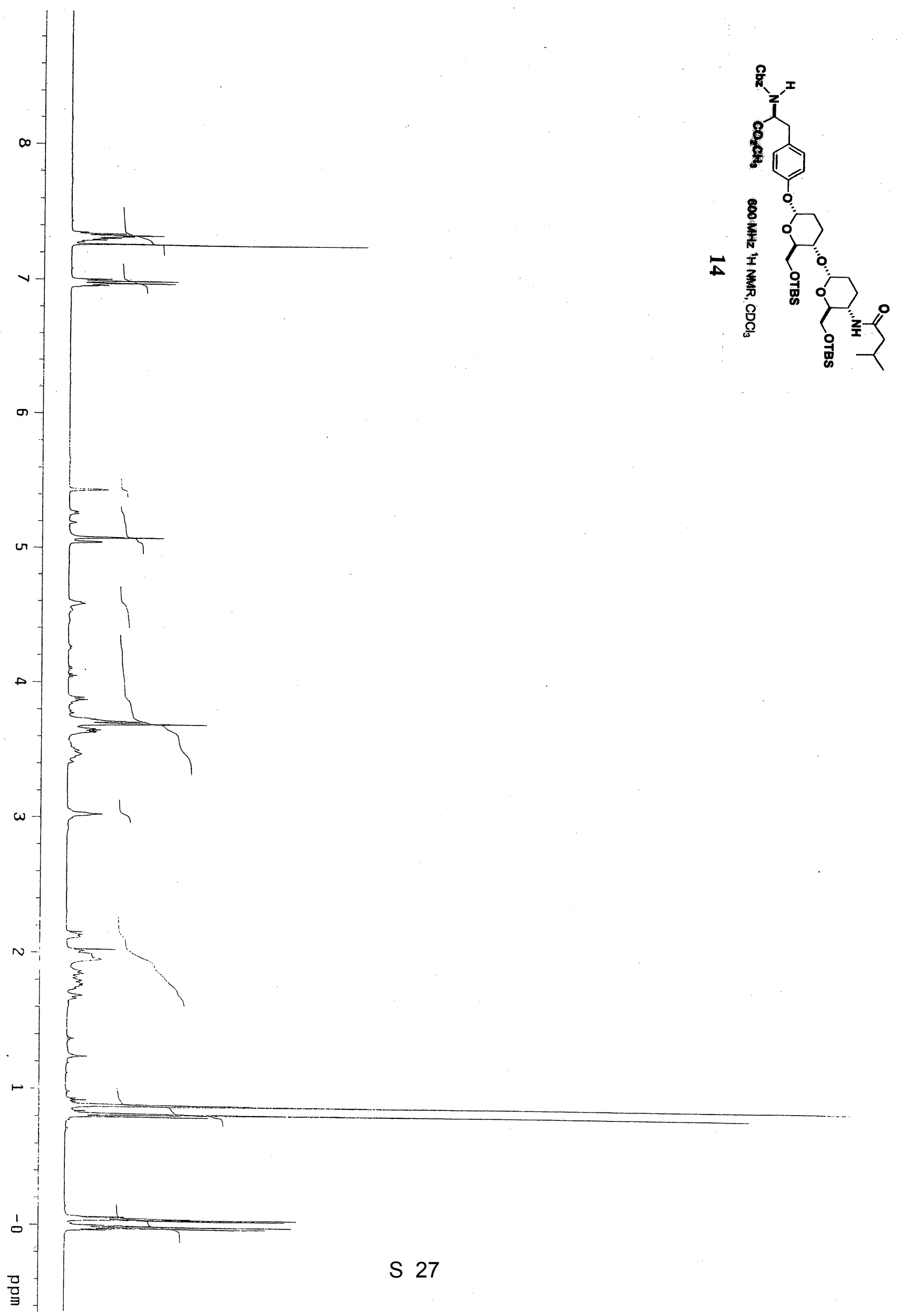




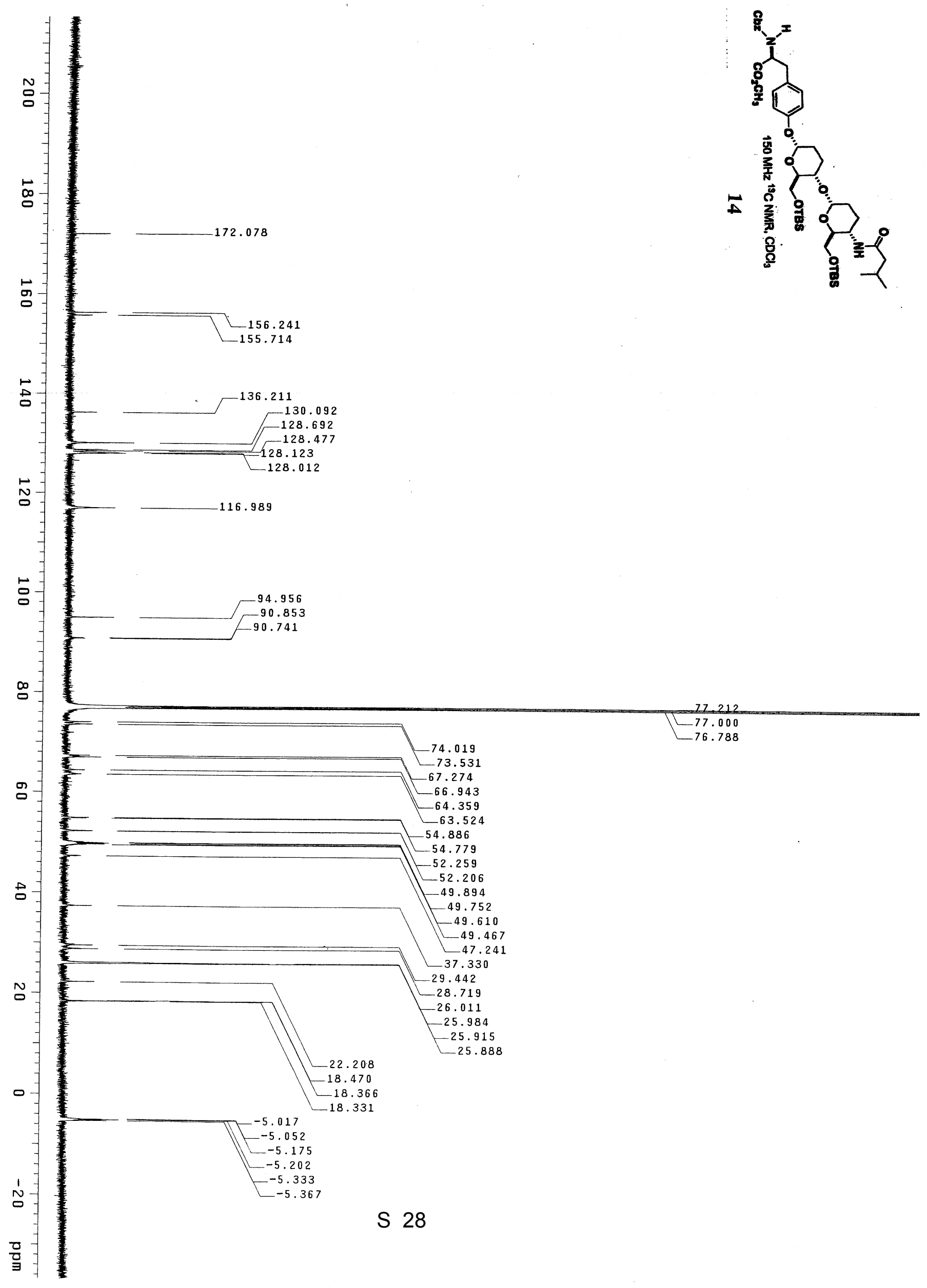

